\title{
Non-local $\mathcal{P} \mathcal{T}$-symmetric potentials in the one-dimensional Dirac equation
}

\author{
Francesco Cannata ${ }^{1}$ and Alberto Ventura ${ }^{2}$ \\ ${ }^{1}$ Istituto Nazionale di Fisica Nucleare, Sezione di Bologna, \\ Via Irnerio 46, I-40126 Bologna, Italy \\ 2Ente per le Nuove Tecnologie, l' Energia e l' Ambiente, \\ Via Martiri di Monte Sole 4, I-40129 Bologna, \\ and Istituto Nazionale di Fisica Nucleare, Sezione di Bologna, Italy \\ Francesco.Cannata@bo.infn.it, Alberto.Ventura@bologna.enea.it
}

\begin{abstract}
The Dirac equation in $(1+1)$ dimensions with a non-local $\mathcal{P} \mathcal{T}$ symmetric potential of separable type is studied by means of the Green function method: properties of bound and scattering states are derived in full detail and numerical results are shown for a potential kernel of Yamaguchi type, inspired by the treatment of low-energy nucleonnucleon interaction.

PACS:03.65.CGe, 03.65.Nk, 03.65.Pm, 11.30.Er, 11.55.Ds
\end{abstract}

\section{Introduction}

Since the pioneering papers by Bender and coworkers[1], [2], the study of non-Hermitian Hamiltonians invariant under space-time reflection has developed into a branch of quantum mechanics in its own, called $\mathcal{P} \mathcal{T}$-symmetric quantum mechanics. The large majority of analyses have been devoted to bound state problems, where the observation that $\mathcal{P} \mathcal{T}$-symmetric Hamiltonians with eigenfunctions that are eigenstates of $\mathcal{P} \mathcal{T}$ have real spectra has led to Hermitian-equivalent formulations, where one can define a linear operator 
$\mathcal{C}$, commuting with Hamiltonian $H$ and with $\mathcal{P} \mathcal{T}$, that permits constructing time-independent inner products with positive-definite norms of the form $\int_{-\infty}^{+\infty} \Psi(x) \mathcal{C P} \mathcal{T} \Psi(x) d x$ (see Ref. 3] for a review).

$\mathcal{P} \mathcal{T}$-symmetric quantum mechanics has a close connection with the more general quasi-Hermitian quantum mechanics [4], [5], [6], where $H$ is called quasiHermitian if it satisfies the intertwining relation $H^{\dagger}=\eta_{+} H \eta_{+}^{-1}$, with $\eta_{+}$a positive-definite Hermitian operator called the metric operator, playing a role analogous to $\mathcal{C P}$.

While bound states of $\mathcal{P} \mathcal{T}$-symmetric Hamiltonians are nowadays well understood, many more questions remain open in the treatment of scattering states: for instance, it has been shown in Ref.[7] that even simple local potentials require introducing non-local metric operators and non-standard boundary conditions with progressive and regressive waves not only in the initial but also in the final state. Even if the latter feature might be removed by an appropriate choice of the metric operator, called quasi-local in Refs. [8], [9], a satisfactory general approach has not been formulated yet. This is why the majority of studies on scattering by $\mathcal{P} \mathcal{T}$-symmetric Hamiltonians has been made within the framework of standard quantum mechanics, breaking unitarity of the corresponding scattering matrices (see Ref.[1] and references therein). Even at this effective level, $\mathcal{P} \mathcal{T}$-symmetric potentials are peculiar, in the sense that, depending on their parameters, they may behave as absorptive for progressive waves and generative for regressive ones (or viceversa), a property called handedness in Ref.[10], or it may happen that they are neither absorptive, nor generative, because the sum of the square moduli of transmission and reflection coefficients may be smaller than one, or greater than one in different intervals of incident energy; they can even conserve unitarity when the asymptotic wave functions are eigenstates of $\mathcal{P} \mathcal{T}$ : in this latter case they are necessarily reflectionless[11. As is known, the reflection of progressive (left-to-right) and regressive (right-to-left) waves is quite asymmetric $\left(R_{L \rightarrow R} \neq R_{R \rightarrow L}\right)$ already in the case of local potentials, where the transmission is the same; in the case of non-local potentials [12], the transmission is asymmetric, too $\left(T_{L \rightarrow R} \neq T_{R \rightarrow L}\right)$. Indeed, non-local potentials have more subtle $\mathcal{P} \mathcal{T}$-transformation properties than local potentials, for which $\mathcal{T}$ - invariance and Hermiticity requirements coincide[11].

The scenario is even richer in relativistic quantum mechanics, where, again, the majority of studies have been dedicated to bound states of $\mathcal{P} \mathcal{T}$ symmetric potentials in the Klein-Gordon and Dirac equations in $(1+1)$ space-time dimensions. Limiting ourselves to the Dirac equation, of interest 
to the present work, we may quote the pseudo-supersymmetric description [13], [14] of scalar or pseudo-scalar local potentials with exact, or spontaneously broken $\mathcal{P} \mathcal{T}$ symmetry, the $\mathcal{P} \mathcal{T}$-symmetric version of the generalized Hulthén vector potential[15], the combinations of scalar (position-dependent mass) and vector potentials of Refs.[16], [17], [18], [19].

Making again an effective approach to scattering aspects, we have examined in a recent work 20] the Dirac equation with the time component of a vector potential in the form of a $\mathcal{P} \mathcal{T}$-symmetric square well: when the real depth exceeds $2 m$, with $m$ the particle mass, transmission resonances at negative energies appear as the signature of spontaneous pair production, but become weaker with increasing imaginary depth and negligible beyond the critical value at which real bound states disappear.

In the present work, which extends the non-relativistic results of Refs. [11, [12], we consider a scalar and vector combination of non-local separable potentials in the $(1+1)$-dimensional Dirac equation, aimed in particular at the study of symmetries known in their three-dimensional form as the spin and pseudospin symmetries, the latter being experimentally observed in atomic nuclei - Numerical results will be consistently obtained from the $\mathcal{P} \mathcal{T}$-symmetric version of a solvable potential originally proposed by Yamaguchi for the description of bound and scattering states of the neutron-proton system.

Since this kind of potential has received until now moderate attention within the framework of $\mathcal{P} \mathcal{T}$-symmetric quantum mechanics, and, to our knowledge, no attention at all in its relativistic version, we consider it worthwhile to perform a detailed, albeit effective analysis by means of the Green function method described in Section 2. The scattering matrix is then studied in Section 3 and two non-relativistic limits for the particular choices of the ratio of vector and scalar couplings corresponding to spin and pseudo-spin symmetry are discussed in Section 4. Bound states are studied in Section 5 and numerical results obtained with a kernel corresponding to the Yamaguchi potential are discussed in Section 6. Section 7 is dedicated to conclusions and perspectives of future work. 


\section{Green function approach}

Let us start with the $(1+1)$-dimensional Dirac equation with a vector-plusscalar non-local potential, written in units $\hbar=c=1$

$$
\begin{aligned}
i \frac{\partial}{\partial t} \Psi(x, t) & =\left(-i \alpha_{x} \frac{\partial}{\partial x}+\beta m\right) \Psi(x, t)+\left(c_{S} \beta+c_{V}\right) \int_{-\infty}^{+\infty} d y K(x, y) \Psi(y,(\mathbb{A})) \\
& \equiv\left(\alpha_{x} p_{x}+\beta m\right) \Psi(x, t)+\left(c_{S} \beta+c_{V}\right) \int_{-\infty}^{+\infty} d y K(x, y) \Psi(y, t) \\
& \equiv\left(H_{D} \Psi\right)(x, t) .
\end{aligned}
$$

A stationary wave, $\Psi(x, t)=\Psi(x) e^{-i E t}$, satisfies the equation

$$
\left(H_{D} \Psi\right)(x)=E \Psi(x) .
$$

Here, $\alpha_{x}$ and $\beta$ are $2 \times 2$ anticommuting Dirac matrices with unit square, $\alpha_{x}^{2}=$ $\beta^{2}=\left(\begin{array}{ll}1 & 0 \\ 0 & 1\end{array}\right) \equiv 1_{2}$, which can be identified with two Pauli matrices: in the present work we adopt the Dirac representation[21] $\alpha_{x}=\sigma_{x} \equiv\left(\begin{array}{ll}0 & 1 \\ 1 & 0\end{array}\right)$, $\beta=\sigma_{z} \equiv\left(\begin{array}{cc}1 & 0 \\ 0 & -1\end{array}\right)$, particularly suited to the study of the non-relativistic limit of the model. $c_{S}$ and $c_{V}$ are the real strengths of the scalar potential and of the time component of the vector potential, respectively, with common $\mathcal{P} \mathcal{T}$-symmetric kernel $K(x, y)=K^{*}(-x,-y)$.

Here, as in our previous work 20] on the one-dimensional Dirac equation with a $\mathcal{P} \mathcal{T}$-symmetric square well, we have the parity operator $\mathcal{P}$ in the Dirac representation

$$
\mathcal{P}=e^{i \theta_{\mathcal{P}}} P_{0} \sigma_{z}
$$

where $P_{0}$ changes $x$ into $-x$ and $\theta_{\mathcal{P}}$ is an arbitrary phase factor. In the same representation, the time reversal operator $\mathcal{T}$ reads

$$
\mathcal{T}=e^{i \theta} \mathcal{T} \sigma_{z} \mathcal{K}
$$

where $\mathcal{K}$ performs complex conjugation and $\theta_{\mathcal{T}}$ is an arbitrary phase factor. With the convenient choice $\theta_{\mathcal{T}}=-\theta_{\mathcal{P}}$ the $\mathcal{P} \mathcal{T}$ operator takes the form

$$
\mathcal{P} \mathcal{T}=P_{0} \mathcal{K}
$$


adopted also in non-relativistic quantum mechanics[11], 12].

It is worthwhile to point out that formula (2) does not contain the most general Hamiltonian: for instance, we might add a pseudo-scalar interaction by extending the matrix of coupling strengths to $c_{S} \beta+c_{V}+i c_{P} \alpha_{x} \beta$. The method of solution described in this section could be applied even to the most general case, but we do not consider it explicitly, because we are mainly interested in interaction potentials that permit decoupling the two integrodifferential equations satisfied by the two components of $\Psi$, so as to obtain a clear definition of their non-relativistic limits, as will be shown in detail in Section 4.

In order to deal with a solvable model, we assume a separable kernel of the form

$$
K(x, y)=g(x) e^{i a x} h(y) e^{i b y},
$$

where $a$ and $b$ are real numbers and the real functions $g$ and $h$ are even functions of their arguments, $g(x)=g(-x)$ and $h(y)=h(-y)$, so as to assure $\mathcal{P} \mathcal{T}$ invariance. When $g=h$ and $a=b=0$, the kernel becomes real symmetric and coincides with that of Ref.[22]. When $g=h$ and $a=-b$ the kernel becomes Hermitian, since in that case $K(x, y)=K^{*}(y, x)$.

Now we solve Eq. (3) by means of the Green function method already used for the one-dimensional Schrödinger equation with the same type of potential[11] [12]. The Green function method had already been used in the solution of a scalar-plus-vector real non-local separable potential in Ref. 22. and of a pure vector potential in Ref. [23].

Two linearly independent Green functions, $G_{+}\left(x, x^{\prime}\right)$ and $G_{-}\left(x, x^{\prime}\right)$, for the time-independent Dirac equation (3) are solutions to the equation

$$
\left(-i \alpha_{x} \frac{\partial}{\partial x}+\beta m-(E \pm i \epsilon)\right) G_{ \pm}\left(x, x^{\prime}\right)=\delta\left(x-x^{\prime}\right)
$$

where a small imaginary component $\epsilon(>0)$ is added to the energy, $E$, in order to remove the energy poles from the contour of the complex integral defining $G_{ \pm}\left(x, x^{\prime}\right)$, as discussed in the following part of this Section. $G_{+}\left(x, x^{\prime}\right)$ and $G_{-}\left(x, x^{\prime}\right)$ are related to the Laplace transform with respect to time of the retarded and advanced component of the causal Green function, respectively, as shown in Appendix A.

Eq. (8) is easily solved in momentum space after introducing the Fourier 
transforms $\widetilde{G}_{ \pm}\left(q, q^{\prime}\right)$

$$
G_{ \pm}\left(x, x^{\prime}\right)=\frac{1}{(2 \pi)^{2}} \int_{-\infty}^{+\infty} d q e^{i q x} \int_{-\infty}^{+\infty} d q^{\prime} e^{i q^{\prime} x^{\prime}} \widetilde{G}_{ \pm}\left(q, q^{\prime}\right)
$$

and the Fourier representation of the Dirac $\delta$ function

$$
\delta\left(x-x^{\prime}\right)=\frac{1}{2 \pi} \int_{-\infty}^{+\infty} d q e^{i q\left(x-x^{\prime}\right)} .
$$

After inserting formulae (9-[10) into Eq. (8) we quickly obtain $\widetilde{G}_{ \pm}\left(q, q^{\prime}\right)$ in the form

$$
\begin{aligned}
\widetilde{G}_{ \pm}\left(q, q^{\prime}\right) & =\lim _{\epsilon \rightarrow 0+} 2 \pi\left(\alpha_{x} q+\beta m-E \mp i \epsilon\right)^{-1} \delta\left(q+q^{\prime}\right) \\
& =2 \pi \lim _{\epsilon \rightarrow 0+} \frac{\alpha_{x} q+\beta m+E \pm i \epsilon}{q^{2}+m^{2}-(E \pm i \epsilon)^{2}} \delta\left(q+q^{\prime}\right)
\end{aligned} .
$$

Therefore, we obtain for the Green functions in configuration space

$$
G_{ \pm}\left(x, x^{\prime}\right) \equiv G_{ \pm}\left(x-x^{\prime}\right)=\frac{1}{2 \pi} \lim _{\epsilon \rightarrow 0+} \int_{-\infty}^{+\infty} d q e^{i q\left(x-x^{\prime}\right)} \frac{\alpha_{x} q+\beta m+E \pm i \epsilon}{q^{2}+m^{2}-(E \pm i \epsilon)^{2}}
$$

which can be easily computed by the method of residues. Let us start with $G_{+}\left(x-x^{\prime}\right)$ : after defining $k^{2}=E^{2}-m^{2}$, we observe that the integrand has two simple poles at $q_{1}=-k-i \epsilon^{\prime}$ and $q_{2}=+k+i \epsilon^{\prime}$, where $\epsilon^{\prime}=\epsilon E / k$. For $x-x^{\prime} \geq 0$ the integration contour is closed in the upper $q$ half-plane, including the pole at $q=q_{2}$, while for $x-x^{\prime}<0$ the contour is closed in the lower $q$ half-plane, including the pole at $q=q_{1}$ with a global - sign, because the integration is done in the clockwise direction. The result is

$$
\begin{gathered}
G_{+}\left(x-x^{\prime}\right)=\frac{i}{2 k}\left[\theta\left(x-x^{\prime}\right) e^{i k\left(x-x^{\prime}\right)}\left(\alpha_{x} k+\beta m+E\right)\right. \\
\left.\quad+\theta\left(x^{\prime}-x\right) e^{-i k\left(x-x^{\prime}\right)}\left(-\alpha_{x} k+\beta m+E\right)\right] \\
=\frac{i}{2 k} e^{i k\left|x-x^{\prime}\right|}\left(k \alpha_{x} \operatorname{sgn}\left(x-x^{\prime}\right)+\beta m+E\right),
\end{gathered}
$$

in agreement with Ref.[22]. In the same way we compute $G_{-}\left(x-x^{\prime}\right)$, after observing that for $x-x^{\prime} \geq 0$ the integration contour in the upper $q$ half-plane now includes a pole at $q_{3}=-k+i \epsilon^{\prime}$ while, for $x-x^{\prime}<0$, the contour is closed in the clockwise direction in the lower $q$ half-plane around a pole at $q_{4}=+k-i \epsilon^{\prime}$. The result is

$$
\begin{gathered}
G_{-}\left(x-x^{\prime}\right)=-\frac{i}{2 k}\left[\theta\left(x-x^{\prime}\right) e^{-i k\left(x-x^{\prime}\right)}\left(-\alpha_{x} k+\beta m+E\right)\right. \\
\left.+\theta\left(x^{\prime}-x\right) e^{i k\left(x-x^{\prime}\right)}\left(\alpha_{x} k+\beta m+E\right)\right] \\
=-\frac{i}{2 k} e^{-i k\left|x-x^{\prime}\right|}\left(-k \alpha_{x} \operatorname{sgn}\left(x-x^{\prime}\right)+\beta m+E\right) .
\end{gathered}
$$


Summing up

$$
G_{ \pm}\left(x-x^{\prime}\right)= \pm \frac{i}{2 k} e^{ \pm i k\left|x-x^{\prime}\right|}\left( \pm k \alpha_{x} \operatorname{sgn}\left(x-x^{\prime}\right)+\beta m+E\right)
$$

It is immediate to check that

$$
G_{-}\left(x-x^{\prime}\right)=\mathcal{P} \mathcal{T} G_{+}\left(x-x^{\prime}\right)(\mathcal{P} \mathcal{T})^{-1}
$$

\section{Scattering matrix}

By exploiting the results of the preceding section, we can define two linearly independent solutions to Eq. (3),$\Psi_{+}(x)$ and $\Psi_{-}(x)$, in the implicit form

$$
\begin{aligned}
& \Psi_{ \pm}(x)=\Psi_{\text {free }}^{ \pm}(x)-\int_{-\infty}^{+\infty} d x^{\prime} G_{ \pm}\left(x-x^{\prime}\right)\left(c_{S} \beta+c_{V}\right) \int_{-\infty}^{+\infty} d y K\left(x^{\prime}, y\right) \Psi_{ \pm}(y) \\
= & \Psi_{\text {free }}^{ \pm}(x)-\int_{-\infty}^{+\infty} d x^{\prime} G_{ \pm}\left(x-x^{\prime}\right)\left(c_{S} \beta+c_{V}\right) g\left(x^{\prime}\right) e^{i a x^{\prime}} \int_{-\infty}^{+\infty} d y h(y) e^{i b y} \Psi_{ \pm}(y) .
\end{aligned}
$$

In Eq. (16),$\Psi_{\text {free }}^{ \pm}(x)$ is the general solution to the Dirac equation for a free particle, conveniently written in the matrix notation of Ref.[21]

$$
\Psi_{f r e e}^{ \pm}(x)=\left(\begin{array}{cc}
e^{i k x} & e^{-i k x} \\
\lambda e^{i k x} & -\lambda e^{-i k x}
\end{array}\right) \cdot\left(\begin{array}{c}
A_{ \pm} \\
B_{ \pm}
\end{array}\right)=\left(\begin{array}{c}
A_{ \pm} e^{i k x}+B_{ \pm} e^{-i k x} \\
\lambda A_{ \pm} e^{i k x}-\lambda B_{ \pm} e^{-i k x}
\end{array}\right)
$$

where $\lambda \equiv k /(E+m)=\sqrt{(E-m) /(E+m)}$ and $A_{ \pm}$and $B_{ \pm}$are arbitrary constants. It is worthwhile to point out that $G_{ \pm}\left(x-x^{\prime}\right)$ and $c_{S} \beta+c_{V}$ are non-commuting $2 \times 2$ matrices: therefore, their order is not arbitrary.

After defining $I_{ \pm} \equiv \int_{-\infty}^{+\infty} d y h(y) e^{i b y} \Psi_{ \pm}(y)$, we multiply both sides of Eq. (16) by $h(x) e^{i b x}$ and integrate them over $x$ from $-\infty$ to $+\infty$. Remembering that $\widetilde{f}(q)=\int_{-\infty}^{+\infty} d x e^{-i q x} f(x)$ is the Fourier transform of $f(x)$ and observing that $f(x)=f(-x)$ implies $\tilde{f}(q)=\tilde{f}(-q)$, we promptly obtain

$$
\begin{gathered}
I_{ \pm}=\left(\begin{array}{c}
A_{ \pm} \widetilde{h}(k+b)+B_{ \pm} \widetilde{h}(k-b) \\
\lambda A_{ \pm} \widetilde{h}(k+b)-\lambda B_{ \pm} \widetilde{h}(k-b)
\end{array}\right) \\
-\int_{-\infty}^{+\infty} d x h(x) e^{i b x} \int_{-\infty}^{+\infty} d x^{\prime} G_{ \pm}\left(x-x^{\prime}\right)\left(c_{S} \beta+c_{V}\right) g\left(x^{\prime}\right) e^{i a x^{\prime}} I_{ \pm} \\
=\left(\begin{array}{c}
A_{ \pm} \widetilde{h}(k+b)+B_{ \pm} \widetilde{h}(k-b) \\
\lambda A_{ \pm} \widetilde{h}(k+b)-\lambda B_{ \pm} \widetilde{h}(k-b)
\end{array}\right)-N_{ \pm}\left(c_{S} \beta+c_{V}\right) I_{ \pm},
\end{gathered}
$$


where $N_{ \pm} \equiv \int_{-\infty}^{+\infty} d x h(x) e^{i b x} \int_{-\infty}^{+\infty} d x^{\prime} G_{ \pm}\left(x-x^{\prime}\right) g\left(x^{\prime}\right) e^{i a x^{\prime}}$. Therefore, the spinor $I_{ \pm}$is explicitly given by the relation

$$
I_{ \pm}=\left(1_{2}+N_{ \pm}\left(c_{S} \beta+c_{V}\right)\right)^{-1} \cdot\left(\begin{array}{c}
A_{ \pm} \widetilde{h}(k+b)+B_{ \pm} \widetilde{h}(k-b) \\
\lambda A_{ \pm} \widetilde{h}(k+b)-\lambda B_{ \pm} \widetilde{h}(k-b)
\end{array}\right)
$$

once we have determined the $2 \times 2$ matrix $N_{ \pm}$, which, according to formula (15), is conveniently rewritten as

$$
\begin{gathered}
N_{ \pm}= \pm \frac{i}{2 k} \int_{-\infty}^{+\infty} d x h(x) e^{i b x} \int_{-\infty}^{+\infty} d x^{\prime} g\left(x^{\prime}\right) e^{i a x^{\prime}}\left[e^{ \pm i k\left(x-x^{\prime}\right)}\left( \pm \alpha_{x} k+\beta m+E\right) \theta\left(x-x^{\prime}\right)\right. \\
\left.\quad+e^{\mp i k\left(x-x^{\prime}\right)}\left(\mp \alpha_{x} k+\beta m+E\right) \theta\left(x^{\prime}-x\right)\right] \\
= \pm \frac{i}{2 k}\left[N_{ \pm}^{(1)}\left( \pm \alpha_{x} k+\beta m+E\right)+N_{ \pm}^{(2)}\left(\mp \alpha_{x} k+\beta m+E\right)\right]
\end{gathered}
$$

where

$$
N_{ \pm}^{(1)}(a, b, k) \equiv \int_{-\infty}^{+\infty} d x h(x) e^{i b x} \int_{-\infty}^{+\infty} d x^{\prime} g\left(x^{\prime}\right) e^{i a x^{\prime}} e^{ \pm i k\left(x-x^{\prime}\right)} \theta\left(x-x^{\prime}\right)
$$

and

$$
N_{ \pm}^{(2)}(a, b, k) \equiv \int_{-\infty}^{+\infty} d x h(x) e^{i b x} \int_{-\infty}^{+\infty} d x^{\prime} g\left(x^{\prime}\right) e^{i a x^{\prime}} e^{\mp i k\left(x-x^{\prime}\right)} \theta\left(x^{\prime}-x\right) .
$$

It is worthwhile to point out the following symmetry relation

$$
N_{+}^{(j)}(-a,-b, k)=\left(N_{-}^{(j)}(a, b, k)\right)^{*}(j=1,2) .
$$

After introducing the linear combinations

$S_{ \pm}(a, b, k) \equiv N_{ \pm}^{(1)}(a, b, k)+N_{ \pm}^{(2)}(a, b, k), \quad D_{ \pm}(a, b, k)=N_{ \pm}^{(1)}(a, b, k)-N_{ \pm}^{(2)}(a, b, k)$ with symmetry relations

$$
S_{+}(-a,-b, k)=\left(S_{-}(a, b, k)\right)^{*}, D_{+}(-a,-b, k)=\left(D_{-}(a, b, k)\right)^{*},
$$

$N_{ \pm}$becomes

$$
N_{ \pm}=\frac{i}{2 k}\left[D_{ \pm} \alpha_{x} k \pm S_{ \pm}(\beta m+E)\right]
$$


We now specialize to the Dirac representation, already introduced in Section $2, \alpha_{x}=\sigma_{x}, \beta=\sigma_{z}$. After some simple algebra, we obtain

$$
\begin{gathered}
N_{ \pm}=\left(\begin{array}{cc} 
\pm i \frac{S_{ \pm}}{2 \lambda} & i \frac{D_{ \pm}}{2} \\
i \frac{D_{ \pm}}{2} & \pm i \frac{S_{ \pm}}{2}
\end{array}\right), \\
M_{ \pm} \equiv 1_{2}+N_{ \pm}\left(c_{S} \beta+c_{V}\right) \\
=\left(\begin{array}{cc}
1 \pm \frac{i}{2} \frac{S_{ \pm}}{\lambda}\left(c_{V}+c_{S}\right) & \frac{i}{2} D_{ \pm}\left(c_{V}-c_{S}\right) \\
\frac{i}{2} D_{ \pm}\left(c_{V}+c_{S}\right) & 1 \pm \frac{i}{2} \lambda S_{ \pm}\left(c_{V}-c_{S}\right)
\end{array}\right) .
\end{gathered}
$$

In order to compute $I_{ \pm}$from formula (19), we need the inverse of $M_{ \pm}$

$$
M_{ \pm}^{-1}=\frac{1}{\operatorname{det} M_{ \pm}}\left(\begin{array}{cc}
1 \pm \frac{i}{2} \lambda S_{ \pm}\left(c_{V}-c_{S}\right) & -\frac{i}{2} D_{ \pm}\left(c_{V}-c_{S}\right) \\
-\frac{i}{2} D_{ \pm}\left(c_{V}+c_{S}\right) & 1 \pm \frac{i}{2} \frac{S_{ \pm}}{\lambda}\left(c_{V}+c_{S}\right)
\end{array}\right),
$$

with

$$
\begin{gathered}
\operatorname{det} M_{ \pm}=\left(1 \pm \frac{i}{2} \lambda S_{ \pm}\left(c_{V}-c_{S}\right)\right)\left(1 \pm \frac{i}{2} \frac{S_{ \pm}}{\lambda}\left(c_{V}+c_{S}\right)\right)+\frac{D_{ \pm}^{2}}{4}\left(c_{V}^{2}-c_{S}^{2}\right) \\
=1 \pm \frac{i}{2} S_{ \pm}\left(\lambda\left(c_{V}-c_{S}\right)+\frac{1}{\lambda}\left(c_{V}+c_{S}\right)\right)+\frac{c_{V}^{2}-c_{S}^{2}}{4}\left(D_{ \pm}^{2}-S_{ \pm}^{2}\right) \\
=1 \pm i \frac{S_{ \pm}}{k}\left(c_{V} E+c_{S} m\right)+\frac{c_{V}^{2}-c_{S}^{2}}{4}\left(D_{ \pm}^{2}-S_{ \pm}^{2}\right)
\end{gathered}
$$

Note that, as a consequence of relations (23, 25),

$$
\operatorname{det} M_{-}(-a,-b, k)=\left(\operatorname{det} M_{+}(a, b, k)\right)^{*} \text {. }
$$

We are now in a position to express the asymptotic forms of the wave functions $\Psi_{ \pm}(x)$ and the transmission and reflection coefficients for progressive and regressive waves in terms of known quantities. For the sake of clarity, let us consider $\Psi_{+}(x)$ and $\Psi_{-}(x)$ separately.

In order to determine the asymptotic behaviour of $\Psi_{+}(x)$, we observe that

$$
\lim _{x \rightarrow \pm \infty} G_{+}\left(x-x^{\prime}\right)=\frac{i}{2} e^{ \pm i k\left(x-x^{\prime}\right)}\left(\begin{array}{cc}
\frac{1}{\lambda} & \pm 1 \\
\pm 1 & \lambda
\end{array}\right) .
$$

Therefore, in particular

$$
\begin{gathered}
\lim _{x \rightarrow+\infty} \Psi_{+}(x)=A_{+}\left(\begin{array}{c}
1 \\
\lambda
\end{array}\right) e^{i k x}+B_{+}\left(\begin{array}{c}
1 \\
-\lambda
\end{array}\right) e^{-i k x} \\
-\frac{i}{2} \int_{-\infty}^{+\infty} d x^{\prime} e^{i k\left(x-x^{\prime}\right)} e^{i a x^{\prime}} g\left(x^{\prime}\right)\left(\begin{array}{cc}
\frac{1}{\lambda} & 1 \\
1 & \lambda
\end{array}\right) \cdot\left(\begin{array}{cc}
c_{V}+c_{S} & 0 \\
0 & c_{V}-c_{S}
\end{array}\right) \cdot I_{+} \\
=A_{+}\left(\begin{array}{c}
1 \\
\lambda
\end{array}\right) e^{i k x}+B_{+}\left(\begin{array}{c}
1 \\
-\lambda
\end{array}\right) e^{-i k x} \\
-\frac{i}{2} \widetilde{g}(a-k)\left(\begin{array}{cc}
\frac{c_{V}+c_{S}}{\lambda} & c_{V}-c_{S} \\
c_{V}+c_{S} & \lambda\left(c_{V}-c_{S}\right)
\end{array}\right) \cdot I_{+} e^{i k x}
\end{gathered}
$$


If we impose the condition that $\Psi_{+}(x)$ is a progressive wave, travelling from left to right $(L \rightarrow R)$, we can put $A_{+}=1$ and $B_{+}=0$ in the preceding equation. After deriving from formula (19) the explicit form of $I_{+}$

$$
I_{+}=\frac{\widetilde{h}(k+b)}{\operatorname{det} M_{+}}\left(\begin{array}{c}
1+\frac{i}{2} \lambda S_{+}\left(c_{V}-c_{S}\right)-\frac{i}{2} \lambda D_{+}\left(c_{V}-c_{S}\right) \\
-\frac{i}{2} D_{+}\left(c_{V}+c_{S}\right)+\lambda+\frac{i}{2} S_{+}\left(c_{V}+c_{S}\right)
\end{array}\right),
$$

the above limit can be rewritten after some algebra in the form

$$
\begin{gathered}
\lim _{x \rightarrow+\infty} \Psi_{+}(x)=\left(\begin{array}{c}
1 \\
\lambda
\end{array}\right) e^{i k x}\left[1-\frac{i}{2} \widetilde{g}(a-k) \widetilde{h}(k+b) \times\right. \\
\left.\frac{\frac{2}{k}\left(c_{V} E+c_{S} m\right)+i\left(c_{V}^{2}-c_{S}^{2}\right)\left(S_{+}-D_{+}\right)}{1+\frac{S_{+}}{k}\left(c_{V} E+c_{S} m\right)+\frac{1}{4}\left(c_{V}^{2}-c_{S}^{2}\right)\left(D_{+}^{2}-S_{+}^{2}\right)}\right]
\end{gathered}
$$

allowing us to determine the transmission coefficient, $T_{L \rightarrow R}$, since we must have

$$
\lim _{x \rightarrow+\infty} \Psi_{+}(x)=T_{L \rightarrow R}\left(\begin{array}{c}
1 \\
\lambda
\end{array}\right) e^{i k x} .
$$

From comparison of the r.h.s. of Eqs (35) and (36) we obtain

$T_{L \rightarrow R}=1-\frac{i}{2} \widetilde{g}(a-k) \widetilde{h}(k+b) \frac{\frac{2}{k}\left(c_{V} E+c_{S} m\right)+i\left(c_{V}^{2}-c_{S}^{2}\right)\left(S_{+}-D_{+}\right)}{1+i \frac{S_{+}}{k}\left(c_{V} E+c_{S} m\right)+\frac{1}{4}\left(c_{V}^{2}-c_{S}^{2}\right)\left(D_{+}^{2}-S_{+}^{2}\right)}$.

In the same way we can compute the reflection coefficient, $R_{L \rightarrow R}$, starting from

$$
\begin{gathered}
\lim _{x \rightarrow-\infty} \Psi_{+}(x)=\left(\begin{array}{c}
1 \\
\lambda
\end{array}\right) e^{i k x} \\
-\frac{i}{2} e^{-i k x} \int_{-\infty}^{+\infty} d x^{\prime} e^{i(k+a) x^{\prime}} g\left(x^{\prime}\right)\left(\begin{array}{cc}
\frac{1}{\lambda} & -1 \\
-1 & \lambda
\end{array}\right) \cdot\left(\begin{array}{cc}
c_{V}+c_{S} & 0 \\
0 & c_{V}-c_{S}
\end{array}\right) \cdot I_{+} \\
=\left(\begin{array}{c}
1 \\
\lambda
\end{array}\right) e^{i k x}-\frac{i}{2} e^{-i k x} \widetilde{g}(k+a)\left(\begin{array}{cc}
\frac{c_{V}+c_{S}}{\lambda} & c_{S}-c_{V} \\
-\left(c_{V}+c_{S}\right) & \lambda\left(c_{V}-c_{S}\right)
\end{array}\right) \cdot I_{+} .
\end{gathered}
$$

Using again formula (34) for $I_{+}$, we obtain after some simple algebra

$$
\lim _{x \rightarrow-\infty} \Psi_{+}(x)=\left(\begin{array}{c}
1 \\
\lambda
\end{array}\right) e^{i k x}-\frac{i}{2} \frac{\widetilde{g}(k+a) \widetilde{h}(k+b)}{\operatorname{det} M_{+}}\left(\frac{c_{V}+c_{S}}{\lambda}+\lambda\left(c_{S}-c_{V}\right)\right)\left(\begin{array}{c}
1 \\
-\lambda
\end{array}\right) e^{-i k x},
$$


where det $M_{+}$is given by formula (30). On the other hand, we must have

$$
\lim _{x \rightarrow-\infty} \Psi_{+}(x)=\left(\begin{array}{l}
1 \\
\lambda
\end{array}\right) e^{i k x}+R_{L \rightarrow R}\left(\begin{array}{c}
1 \\
-\lambda
\end{array}\right) e^{-i k x} .
$$

From formulae (39) and (40) we promptly obtain

$$
R_{L \rightarrow R}=-\frac{i}{k} \widetilde{g}(k+a) \widetilde{h}(k+b) \frac{c_{V} m+c_{S} E}{1+i \frac{S_{+}}{k}\left(c_{V} E+c_{S} m\right)+\frac{1}{4}\left(c_{V}^{2}-c_{S}^{2}\right)\left(D_{+}^{2}-S_{+}^{2}\right)} .
$$

In order to compare our results with those of Ref.[22] for a real symmetric kernel, with $g(x)=h(x) \equiv v(x)$ and $a=b=0$, we observe that, in this limit, $D_{+}=0$ and $S_{+} \equiv J=\int_{-\infty}^{+\infty} d x \int_{-\infty}^{+\infty} d x^{\prime} e^{i k\left|x-x^{\prime}\right|} v(x) v\left(x^{\prime}\right) . \quad J$ is promptly expressed in terms of the Fourier transform of $v(x), \widetilde{v}(k) \equiv$ $\int_{-\infty}^{+\infty} d x v(x) e^{-i k x}$. In fact

$$
J=J_{R}+i J_{I}=\int_{-\infty}^{+\infty} d x \int_{-\infty}^{+\infty} d x^{\prime} v(x) v\left(x^{\prime}\right)\left[\cos k\left|x-x^{\prime}\right|+i \sin k\left|x-x^{\prime}\right|\right]
$$

where

$$
\begin{gathered}
J_{R}=\int_{-\infty}^{+\infty} d x \int_{-\infty}^{+\infty} d x^{\prime} v(x) v\left(x^{\prime}\right) \cos k\left(x-x^{\prime}\right) \\
=\int_{-\infty}^{+\infty} d x \int_{-\infty}^{+\infty} d x^{\prime} v(x) v\left(x^{\prime}\right) e^{i k\left(x-x^{\prime}\right)}=\widetilde{v}(k) \widetilde{v}(-k)=(\widetilde{v}(k))^{2}
\end{gathered}
$$

and

$$
i J_{I}=J-J_{R}=J-(\widetilde{v}(k))^{2} .
$$

Thus, in the same limit, we obtain $S_{+}-\widetilde{g}(k) \widetilde{h}(k)=J-(\widetilde{v}(k))^{2}=$ $J-J_{R}=i J_{I}$ and $S_{+}^{2}-2 \widetilde{g}(k) \widetilde{h}(k) S_{+}=J\left(J-2 J_{R}\right)=-J J^{*}=-|J|^{2}$. Therefore, our $\left|T_{L \rightarrow R}\right|^{2}$, from formula (37), coincides with formula (13) of Ref.[22] and our $\left|R_{L \rightarrow R}\right|^{2}$, from formula (41), with formula (14) of the same reference, as expected.

Let us now consider the second Green function, $G_{-}\left(x-x^{\prime}\right)$, whose asymptotic behaviour is

$$
\lim _{x \rightarrow \pm \infty} G_{-}\left(x-x^{\prime}\right)=-\frac{i}{2} e^{\mp i k\left(x-x^{\prime}\right)}\left(\begin{array}{cc}
\frac{1}{\lambda} & \mp 1 \\
\mp 1 & \lambda
\end{array}\right) .
$$


Therefore, in particular

$$
\begin{gathered}
\lim _{x \rightarrow-\infty} \Psi_{-}(x)=A_{-}\left(\begin{array}{c}
1 \\
\lambda
\end{array}\right) e^{i k x}+B_{-}\left(\begin{array}{c}
1 \\
-\lambda
\end{array}\right) e^{-i k x} \\
+\frac{i}{2} \int_{-\infty}^{+\infty} d x^{\prime} e^{i k\left(x-x^{\prime}\right)} e^{i a x^{\prime}} g\left(x^{\prime}\right)\left(\begin{array}{cc}
\frac{1}{\lambda} & 1 \\
1 & \lambda
\end{array}\right) \cdot\left(\begin{array}{cc}
c_{S}+c_{V} & 0 \\
0 & c_{V}-c_{S}
\end{array}\right) \cdot I_{-} \\
=\left[A_{-}\left(\begin{array}{c}
1 \\
\lambda
\end{array}\right)+\frac{i}{2} \widetilde{g}(a-k)\left(\begin{array}{cc}
\frac{c_{S}+c_{V}}{\lambda} & c_{V}-c_{S} \\
c_{S}+c_{V} & \lambda\left(c_{V}-c_{S}\right)
\end{array}\right) \cdot I_{-}\right] e^{i k x}+B_{-}\left(\begin{array}{c}
1 \\
-\lambda
\end{array}\right) e^{-i k x} .
\end{gathered}
$$

We can impose the condition that $\Psi_{-}(x)$ is a regressive wave, travelling from right to left $(R \rightarrow L)$, so that

$$
\lim _{x \rightarrow-\infty} \Psi_{-}(x)=T_{R \rightarrow L}\left(\begin{array}{c}
1 \\
-\lambda
\end{array}\right) e^{-i k x} .
$$

Comparison of formulae (46) and (47) yields

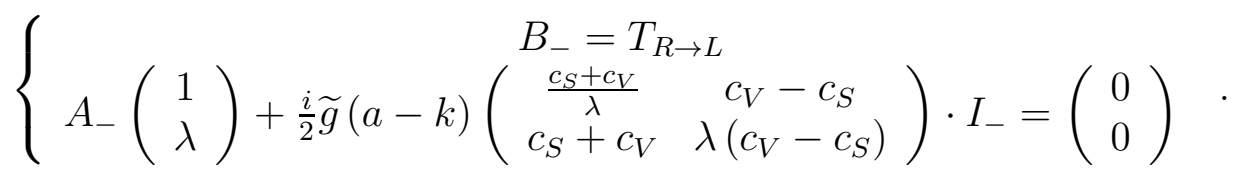

In the same way

$$
\begin{gathered}
\lim _{x \rightarrow+\infty} \Psi_{-}(x)=A_{-}\left(\begin{array}{c}
1 \\
\lambda
\end{array}\right) e^{i k x}+B_{-}\left(\begin{array}{c}
1 \\
-\lambda
\end{array}\right) e^{-i k x} \\
+\frac{i}{2} \int_{-\infty}^{+\infty} d x^{\prime} e^{-i k\left(x-x^{\prime}\right)} e^{i a x^{\prime}} g\left(x^{\prime}\right)\left(\begin{array}{cc}
\frac{1}{\lambda} & -1 \\
-1 & \lambda
\end{array}\right) \cdot\left(\begin{array}{cc}
c_{S}+c_{V} & 0 \\
0 & c_{V}-c_{S}
\end{array}\right) \cdot I_{-} \\
=A_{-}\left(\begin{array}{c}
1 \\
\lambda
\end{array}\right) e^{i k x}+\left[B_{-}\left(\begin{array}{c}
1 \\
-\lambda
\end{array}\right)+\frac{i}{2} \widetilde{g}(a+k)\left(\begin{array}{cc}
\frac{c_{S}+c_{V}}{\lambda} & c_{S}-c_{V} \\
-\left(c_{S}+c_{V}\right) & \lambda\left(c_{V}-c_{S}\right)
\end{array}\right) \cdot I_{-}\right] e^{-i k x} .
\end{gathered}
$$

Since we know that

$$
\lim _{x \rightarrow+\infty} \Psi_{-}(x)=\left(\begin{array}{c}
1 \\
-\lambda
\end{array}\right) e^{-i k x}+R_{R \rightarrow L}\left(\begin{array}{c}
1 \\
\lambda
\end{array}\right) e^{i k x},
$$

we obtain

$$
\left\{\begin{array}{c}
A_{-}=R_{R \rightarrow L} \\
B_{-}\left(\begin{array}{c}
1 \\
-\lambda
\end{array}\right)+\frac{i}{2} \widetilde{g}(a+k)\left(\begin{array}{cc}
\frac{c_{S}+c_{V}}{\lambda} & c_{S}-c_{V} \\
-\left(c_{S}+c_{V}\right) & \lambda\left(c_{V}-c_{S}\right)
\end{array}\right) \cdot I_{-}=\left(\begin{array}{c}
1 \\
-\lambda
\end{array}\right) .
\end{array}\right.
$$


Remembering the expression of $I_{-}$from formulae (19, 29, 30 ), and rewriting it more compactly as

$$
I_{-}=\frac{1}{\operatorname{det} M_{-}}\left(\begin{array}{cc}
1-\frac{i}{2} \lambda S_{-}\left(c_{V}-c_{S}\right) & -\frac{i}{2} \lambda D_{-}\left(c_{V}-c_{S}\right) \\
-\frac{i}{2} D_{-}\left(c_{V}+c_{S}\right) & 1-\frac{i}{2} \frac{S_{-}}{\lambda}\left(c_{V}+c_{S}\right)
\end{array}\right) \cdot\left(\begin{array}{c}
\mathfrak{S} \\
\lambda \mathfrak{D}
\end{array}\right)
$$

with $\mathfrak{S} \equiv A_{-} \widetilde{h}(k+b)+B_{-} \widetilde{h}(k-b)=R_{R \rightarrow L} \widetilde{h}(k+b)+T_{R \rightarrow L} \widetilde{h}(k-b)$ and $\mathfrak{D} \equiv R_{R \rightarrow L} \widetilde{h}(k+b)-T_{R \rightarrow L} \widetilde{h}(k-b)$, we obtain from Eqs. (48,51) a system of two linear equations in the unknowns $\mathfrak{S}$ and $\mathfrak{D}$

$$
\left\{\begin{array}{l}
A_{-}+\frac{i}{2} \frac{\widetilde{g}(a-k)}{\operatorname{det} M_{-}} \mathfrak{P}_{+}=0 \\
B_{-}+\frac{i}{2} \frac{\widetilde{g}(a+k)}{\operatorname{det} M_{-}} \mathfrak{P}_{-}=1
\end{array},\right.
$$

with

$$
\begin{aligned}
\mathfrak{P}_{ \pm}=\left[\frac{c_{S}+c_{V}}{\lambda}-\frac{i}{2}\left(c_{V}^{2}-c_{S}^{2}\right)\left(S_{-}\right.\right. & \left.\left. \pm D_{-}\right)\right] \mathfrak{S} \pm\left[\lambda\left(c_{V}-c_{S}\right)-\frac{i}{2}\left(c_{V}^{2}-c_{S}^{2}\right)\left(S_{-} \pm D_{-}\right)\right] \mathfrak{D} \\
& \equiv \mathfrak{P}_{ \pm}^{(\mathfrak{S})} \mathfrak{S}+\mathfrak{P}_{ \pm}^{(\mathfrak{D})} \mathfrak{D},
\end{aligned}
$$

where

$$
\begin{gathered}
\mathfrak{P}_{ \pm}^{(\mathfrak{S})} \equiv\left[\frac{c_{S}+c_{V}}{\lambda}-\frac{i}{2}\left(c_{V}^{2}-c_{S}^{2}\right)\left(S_{-} \pm D_{-}\right)\right] \\
\mathfrak{P}_{ \pm}^{(\mathfrak{D})} \equiv \pm\left[\lambda\left(c_{V}-c_{S}\right)-\frac{i}{2}\left(c_{V}^{2}-c_{S}^{2}\right)\left(S_{-} \pm D_{-}\right)\right]
\end{gathered}
$$

The transmission and reflection coefficients are thus obtained by solving the system (53)

$$
\begin{gathered}
T_{R \rightarrow L}=\frac{\mathfrak{S}-\mathfrak{D}}{2 \widetilde{h}(k-b)}=\frac{\operatorname{det} M_{-}\left(2 \operatorname{det} M_{-}+i \widetilde{g}(a-k) \widetilde{h}(k+b)\left(\mathfrak{P}_{+}^{(\mathfrak{D})}+\mathfrak{P}_{+}^{(\mathfrak{S})}\right)\right)}{d_{\mathfrak{S}}}, \\
R_{R \rightarrow L}=\frac{\mathfrak{S}+\mathfrak{D}}{2 \widetilde{h}(k+b)}=\frac{i \widetilde{g}(a-k) \widetilde{h}(k-b) \operatorname{det} M_{-}\left(\mathfrak{P}_{+}^{(\mathfrak{D})}-\mathfrak{P}_{+}^{(\mathfrak{S})}\right)}{d_{\mathfrak{S}}}
\end{gathered}
$$

with

$$
\begin{gathered}
d_{\mathfrak{S}}=2\left(\operatorname{det} M_{-}\right)^{2}+i \widetilde{g}(a-k) \widetilde{h}(k+b) \operatorname{det} M_{-}\left(\mathfrak{P}_{+}^{(\mathfrak{D})}+\mathfrak{P}_{+}^{(\mathfrak{S})}\right) \\
-i \widetilde{g}(a+k) \widetilde{h}(k-b) \operatorname{det} M_{-}\left(\mathfrak{P}_{-}^{(\mathfrak{D})}-\mathfrak{P}_{-}^{(\mathfrak{S})}\right) \\
+\widetilde{g}(a+k) \widetilde{g}(a-k) \widetilde{h}(k+b) \tilde{h}(k-b)\left(\mathfrak{P}_{+}^{(\mathfrak{S})} \mathfrak{P}_{-}^{(\mathfrak{D})}-\mathfrak{P}_{-}^{(\mathfrak{S})} \mathfrak{P}_{+}^{(\mathfrak{D})}\right)
\end{gathered}
$$

Formulae (56) can be further semplified by noting that

$$
\begin{gathered}
\mathfrak{P}_{+}^{(\mathfrak{D})}+\mathfrak{P}_{+}^{(\mathfrak{S})}=2 \frac{c_{V} E+c_{S} m}{k}-2 i\left(c_{V}^{2}-c_{S}^{2}\right) N_{-}^{(1)}, \\
\mathfrak{P}_{-}^{(\mathfrak{D})}-\mathfrak{P}_{-}^{(\mathfrak{S})}=-2 \frac{c_{V} E+c_{S} m}{k}+2 i\left(c_{V}^{2}-c_{S}^{2}\right) N_{-}^{(2)}, \\
\mathfrak{P}_{+}^{(\mathfrak{S})} \mathfrak{P}_{-}^{(\mathfrak{D})}-\mathfrak{P}_{-}^{(\mathfrak{S})} \mathfrak{P}_{+}^{(\mathfrak{D})}=-2\left(c_{V}^{2}-c_{S}^{2}\right) \operatorname{det} M_{-} .
\end{gathered}
$$


It turns out that

$$
\begin{gathered}
\frac{d_{\mathfrak{S}}}{\operatorname{det} M_{-}}=2\left\{\operatorname{det} M_{-}+i \frac{c_{V} E+c_{S} m}{k}[\widetilde{g}(a-k) \widetilde{h}(k+b)+\widetilde{g}(a+k) \widetilde{h}(k-b)]\right. \\
+\left(c_{V}^{2}-c_{S}^{2}\right)\left[\widetilde{g}(a-k) \widetilde{h}(k+b) N_{-}^{(1)}+\widetilde{g}(a+k) \widetilde{h}(k-b) N_{-}^{(2)}\right. \\
-\widetilde{g}(a+k) \widetilde{g}(a-k) \widetilde{h}(k+b) \widetilde{h}(k-b)]\}=2 \operatorname{det} M_{+} .
\end{gathered}
$$

The last step is proved in detail in Appendix B. With the above result, formulae (56) are written as

$$
\begin{aligned}
T_{R \rightarrow L} & =\frac{\operatorname{det} M_{-}+\widetilde{g}(a-k) \tilde{h}(k+b)\left[i \frac{c_{V} E+c_{S} m}{k}+\left(c_{V}^{2}-c_{S}^{2}\right) N_{-}^{(1)}\right]}{\operatorname{det} M_{+}} \\
R_{R \rightarrow L} & =-\frac{\widetilde{g}(a-k) \widetilde{h}(k-b)\left[i \frac{c_{V} E+c_{S} m}{k}+\left(c_{V}^{2}-c_{S}^{2}\right) N_{-}^{(2)}\right]}{\operatorname{det} M_{+}} .
\end{aligned}
$$

It is straightforward to verify that 12

$$
\begin{aligned}
T_{L \rightarrow R}(-a,-b) & =T_{R \rightarrow L}(a, b), \\
R_{L \rightarrow R}(-a,-b) & =R_{R \rightarrow L}(a, b) .
\end{aligned}
$$

The scattering matrix, $S$, can be defined as in Ref.[11]

$$
S=\left(\begin{array}{cc}
T_{L \rightarrow R} & R_{R \rightarrow L} \\
R_{L \rightarrow R} & T_{R \rightarrow L}
\end{array}\right)
$$

The general properties of the $S$ matrix obtained in Ref.[11] in case of $\mathcal{P}, \mathcal{T}$, or $\mathcal{P} \mathcal{T}$ invariance of the Hamiltonian hold in relativistic quantum mechanics, too. In particular, $\mathcal{P} \mathcal{T}$ symmetry of the Hamiltonian implies

$$
S^{-1}=S^{*}
$$

or

$$
\begin{gathered}
|\operatorname{det} S|=1, \\
\left|T_{L \rightarrow R}\right|=\left|T_{R \rightarrow L}\right|, \\
\operatorname{Im}\left(R_{L \rightarrow R} R_{R \rightarrow L}^{*}\right)=0 .
\end{gathered}
$$

$T_{L \rightarrow R}$ and $T_{R \rightarrow L}$ have the same modulus, but different phase: the latter property, characteristic of non-local potentials, is discussed in particular in Refs.[12],[11].

Finally, the last of conditions (63) implies that $R_{R \rightarrow L}$ and $R_{L \rightarrow R}$ have the same phase, although they have different moduli, since unitarity is broken. 


\section{Symmetries and non-relativistic limits}

Eq. (3) is equivalent to a pair of coupled differential equations in the two components of the Dirac spinor $\Psi(x)=\left(\begin{array}{c}\Psi_{1}(x) \\ \Psi_{2}(x)\end{array}\right)$; in the Dirac representation, where $\alpha_{x}=\sigma_{x}$ and $\beta=\sigma_{z}$

$$
\left\{\begin{array}{c}
(m-E) \Psi_{1}(x)-i \frac{\partial}{\partial x} \Psi_{2}(x)+\left(c_{S}+c_{V}\right) \int_{-\infty}^{+\infty} d y K(x, y) \Psi_{1}(y)=0 \\
-i \frac{\partial}{\partial x} \Psi_{1}(x)-(m+E) \Psi_{2}(x)+\left(c_{V}-c_{S}\right) \int_{-\infty}^{+\infty} d y K(x, y) \Psi_{2}(y)=0
\end{array} .\right.
$$

For arbitrary values of the coupling strengths, $c_{S}$ and $c_{V}$, the above equations do not decouple; decoupling occurs when $c_{V}= \pm c_{S}$. The method of solution described in the preceding section remains valid and the final results for the reflection and transmission coefficients are still given by formulae (37. 41) for progressive waves and by formulae (56) for regressive waves, even if intermediate formulae are different.

In $3+1$ dimensions, the cases $c_{V}=c_{S}$ and $c_{V}=-c_{S}$ are examples of Bell-Ruegg symmetries[24], where the Dirac Hamiltonian commutes with the generators of an $S U(2)$ group, constructed with Dirac matrices and the momentum operator. The eigenstates of the Dirac Hamiltonian belong to the carrier space of the spinor representation of such a group and are thus doubly degenerate. When $c_{V}=c_{S}$, the members of the doublet have the same radial quantum number $n_{r}$, the same orbital momentum $l$ and total angular momentum $j=l \pm \frac{1}{2}$ (spin symmetry). When $c_{V}=-c_{S}$, they have quantum numbers $\left(n_{r}, l, j=l+\underset{2}{\frac{1}{2}}\right)$ and $\left(n_{r}-1, l+2, j=l+\frac{3}{2}\right)$, i.e. the same pseudo-orbital momentum $\widetilde{l}=l+1$ and pseudo-spin $\widetilde{s}=\frac{1}{2}$, so that $j=\widetilde{l} \pm \frac{1}{2}$ (pseudo-spin symmetry). The mean field of heavy nuclei exhibits an approximate pseudo-spin symmetry, experimentally known for many years, but correctly explained as a relativistic effect only few years ago 25. At a phenomenological level, the approximate pseudo-spin symmetry naturally arises in relativistic mean field models, where the nuclear mean field is in practice the sum of an attractive scalar field (the $\sigma$ field) and of a repulsive vector field (the $\omega$ field) of almost the same strength. At a more fundamental level, it can be obtained from sum rules of quantum chromodynamics in nuclear matter[26].

Let us consider the case $c_{V}=c_{S}=c$ first. We promptly obtain in this 
case

$$
\left\{\begin{array}{c}
-\frac{\partial^{2}}{\partial x^{2}} \Psi_{1}(x)+2 c(m+E) \int_{-\infty}^{+\infty} d y K(x, y) \Psi_{1}(y)=\left(E^{2}-m^{2}\right) \Psi_{1}(x) \equiv k^{2} \Psi_{1}(x) \\
\Psi_{2}(x)=\frac{-i}{m+E} \frac{\partial}{\partial x} \Psi_{1}(x)
\end{array} .\right.
$$

The above system is suited to the study of the non-relativistic limit $(E \rightarrow$ $m+\frac{k^{2}}{2 m}$, with $\left.\frac{k^{2}}{2 m} \ll m\right)$, where the first equation of system (65), satisfied by $\Psi_{1}$, becomes a Schrödinger equation with a non-local potential of strength $s=2 c$ and kernel $K . \Psi_{2}$, being proportional to $\frac{\partial}{\partial x} \Psi_{1}$, does not obey a Schrödinger-like equation.

In this limit, the transmission and reflection coefficients obtained in the preceding section simplify considerably. In fact, from formulae (37,41) we promptly obtain, for $c_{V}=c_{S}=c$ and $E \rightarrow m+\frac{k^{2}}{2 m}$

$$
\left\{\begin{array}{c}
\lim _{E \rightarrow m+\frac{k^{2}}{2 m}} T_{L \rightarrow R}=1-i \frac{2 c m}{k} \frac{\widetilde{g}(k-a) \widetilde{h}(k+b)}{1+i \frac{22 m}{k} S_{+}} \\
\lim _{E \rightarrow m+\frac{k^{2}}{2 m}} R_{L \rightarrow R}=-i \frac{2 c m}{k} \frac{\widetilde{g}(k+a) h(k+b)}{1+i \frac{2 c m}{k} S_{+}}
\end{array},\right.
$$

in agreement with formulae (153) of Ref.[11], where $\frac{2 c m}{k}$ is indicated with $\omega$ and $\frac{1}{1+i \frac{2 c m}{k} S_{+}}$with $D_{+}$, not to be confused with the $D_{+}$integral defined in formulae (24) of the preceding section. It is worthwhile to recall that Ref.[11] uses units $2 m=1$, as is common in non-relativistic quantum mechanics.

In the same way, we obtain, after some simple algebra

$$
\left\{\begin{array}{c}
\lim _{E \rightarrow m+\frac{k^{2}}{2 m}} T_{R \rightarrow L}=1-i \frac{2 c m}{k} \frac{\widetilde{g}(k+a) \widetilde{h}(k-b)}{1+i \frac{2 c m}{k}\left[-S_{-}+\widetilde{g}(k-a) \widetilde{h}(k+b)+\widetilde{g}(k+a) \widetilde{h}(k-b)\right]} \\
\lim _{E \rightarrow m+\frac{k^{2}}{2 m}} R_{R \rightarrow L}=-i \frac{2 c m}{k} \frac{\widetilde{g}(k-a) \widetilde{h}(k-b)}{1+i \frac{2 c m}{k}\left[-S_{-}+\widetilde{g}(k-a) \widetilde{h}(k+b)+\widetilde{g}(k+a) \widetilde{h}(k-b)\right]}
\end{array}\right.
$$

which coincide with formulae (156) of Ref.[11], where $i \frac{2 \mathrm{~cm} S_{-}}{k}$ is indicated with $N_{-}$, not to be confused with the $N_{-}$matrix defined in formula (27).

In the case $c_{V}=-c_{S}=c^{\prime} \Psi_{1}$ and $\Psi_{2}$ interchange their role, since the two decoupled equations now are

$$
\left\{\begin{array}{c}
\Psi_{1}(x)=\frac{-i}{E-m} \frac{\partial}{\partial x} \Psi_{2}(x) \\
-\frac{\partial^{2}}{\partial x^{2}} \Psi_{2}(x)+2 c^{\prime}(E-m) \int_{-\infty}^{+\infty} d y K(x, y) \Psi_{2}(y)=\left(E^{2}-m^{2}\right) \Psi_{2}(x) \equiv k^{2} \Psi_{2}(x)
\end{array}\right.
$$

The formulae of transmission and reflection coefficients now depend on $E-m$, to be replaced in the non-relativistic limit by the kinetic energy $\frac{k^{2}}{2 m}$. In that limit, the second equation ([68), satisfied by $\Psi_{2}$, becomes a Schrödinger 
equation with an energy dependent coupling strength $s(k)=c^{\prime} k^{2} /\left(2 m^{2}\right)$, while $\Psi_{1}$ is not solution to a Schrödinger equation.

The final expressions are

$$
\left\{\begin{array}{c}
\lim _{E \rightarrow m+\frac{k^{2}}{2 m}} T_{L \rightarrow R}=1-\frac{i c^{\prime} k}{2 m} \frac{\widetilde{g}(k-a) \widetilde{h}(k+b)}{1+i \frac{c^{\prime} k}{2 m} S_{+}} \\
\lim _{E \rightarrow m+\frac{k^{2}}{2 m}} R_{L \rightarrow R}=i \frac{c^{\prime} k}{2 m} \frac{\widetilde{g}(k+a) \widetilde{h}(k+b)}{1+i \frac{c^{\prime} k}{2 m} S_{+}}
\end{array}\right.
$$

and

$$
\left\{\begin{array}{c}
\lim _{E \rightarrow m+\frac{k^{2}}{2 m}} T_{R \rightarrow L}=1-i \frac{c^{\prime} k}{2 m} \frac{\widetilde{g}(k+a) \widetilde{h}(k-b)}{1+\frac{i c^{\prime} k}{2 m}\left[-S_{-}+\widetilde{g}(k-a) \widetilde{h}(k+b)+\widetilde{g}(k+a) \widetilde{h}(k-b)\right]} \\
\lim _{E \rightarrow m+\frac{k^{2}}{2 m}} R_{R \rightarrow L}=i \frac{c^{\prime} k}{2 m} \frac{\widetilde{g}(k-a) \widetilde{h}(k-b)}{1+\frac{i c^{\prime} k}{2 m}\left[-S_{-}+\widetilde{g}(k-a) \widetilde{h}(k+b)+\widetilde{g}(k+a) \widetilde{h}(k-b)\right]}
\end{array} .\right.
$$

As expected, the above formulae have the same structure as those in the case $c_{V}=c_{S}$, with the constant strength $s=2 c$ replaced with the energydependent strength $s(k)=c^{\prime} k^{2} /\left(2 m^{2}\right)$.

For arbitrary values of $c_{V}$ and $c_{S}$ the equations (64) do not decouple, unless the potential becomes local, $K(x, y)=\delta(x-y) V(x)$. As a consequence, in the particular case of a purely scalar potential, $c_{V}=0$, we do not obtain the pseudo-supersymmetric scheme of Ref.[13], which holds for local potentials only.

Summing up, the cases $c_{V}= \pm c_{S}$, reflecting the Bell-Ruegg symmetries 24] in one dimension, reduce the two-dimensional manifold $\left[\Psi_{1}, \Psi_{2}\right]$ to the onedimensional manifold $\Psi_{1}$ when $c_{V}=c_{S}$, or $\Psi_{2}$ when $c_{V}=-c_{S}$, the latter case being relevant for nuclear physics.

\section{Bound states with real energy}

The $\mathcal{P} \mathcal{T}$ symmetry of the potential kernel, $K$, permits the general statement that either bound state energies are real, or that they come in complex conjugate pairs: in fact, if $\Psi(x)$ is the solution to the stationary Dirac equation with energy $E$ and momentum $p_{x}$

$$
\left(E-\alpha_{x} p_{x}-\beta m\right) \Psi(x)-\left(c_{S} \beta+c_{V}\right) \int_{-\infty}^{+\infty} d y K(x, y) \Psi(y)=0
$$


$\mathcal{P} \mathcal{T} \Psi(x)=\Psi^{*}(-x)$ is solution to the Dirac equation with energy $E^{*}$ and momentum $-p_{x}^{*}$

$$
\left(E^{*}+\alpha_{x} p_{x}^{*}-\beta m\right) \mathcal{P} \mathcal{T} \Psi(x)-\left(c_{S} \beta+c_{V}\right) \int_{-\infty}^{+\infty} d y K^{*}(-x,-y) \mathcal{P} \mathcal{T} \Psi(y)=0 .
$$

We now treat in particular bound states $\Psi_{b s}(x)$ with real energy and imaginary momentum $p_{x}=-p_{x}^{*}$ and investigate the relation between $\Psi_{b s}(x)$ and $\mathcal{P} \mathcal{T} \Psi_{b s}(x)$. From now on, the quantum number $k$ is no more real and positive, as defined in Section 2, but complex.

The Green function formalism permits not only derivation of scattering, but also of bound state wave functions. As is known, bound state energies are located in the interval $-m<E<+m$, where the square of the momentum, $k^{2}=E^{2}-m^{2}$, is negative, $i$. e., $k=i \bar{k}$ is imaginary. Bound state wave functions can be obtained by analytic continuation of one of the two independent scattering solutions, e. $g . \Psi_{+}(x)$ from formula (16), to the positive imaginary $k$ axis, $i$. $e$. we can take $\bar{k}=\sqrt{m^{2}-E^{2}}>0$, and impose the boundary conditions $\lim _{x \rightarrow \pm \infty} \Psi_{+}(x)=0$. Owing to the fact that the Green function $G_{+}$vanishes at $x= \pm \infty$ when $k=i \bar{k}$, we must get rid of the free-wave contribution, by putting $A_{+}=B_{+}=0$. We thus obtain

$$
\Psi_{b s}(x)=-\int_{-\infty}^{+\infty} d x^{\prime} g\left(x^{\prime}\right) e^{i a x^{\prime}} G_{+}\left(x-x^{\prime}\right)\left(c_{S} \sigma_{z}+c_{V}\right) I_{+} . \quad(k=i \bar{k})
$$

Remembering expression (15) for $G_{+}\left(x-x^{\prime}\right), \Psi_{b s}(x)$ can be put in the form

$$
\begin{gathered}
\Psi_{b s}(x)=-\frac{1}{2 \bar{k}}\left\{e^{-\bar{k} x} \mathcal{I}_{1}(x)\left(i \bar{k} \sigma_{x}+m \sigma_{z}+E\right)\right. \\
\left.+e^{\bar{k} x} \mathcal{I}_{2}(x)\left(-i \bar{k} \sigma_{x}+m \sigma_{z}+E\right)\right\}\left(c_{S} \sigma_{z}+c_{V}\right) I_{+},
\end{gathered}
$$

where

$$
\mathcal{I}_{1}(x) \equiv \int_{-\infty}^{x} d x^{\prime} g\left(x^{\prime}\right) e^{(i a+\bar{k}) x^{\prime}}
$$

and

$$
\mathcal{I}_{2}(x) \equiv \int_{x}^{+\infty} d x^{\prime} g\left(x^{\prime}\right) e^{(i a-\bar{k}) x^{\prime}} .
$$

The normalizability of $\Psi_{b s}$ is easily cheched by noting that $\lim _{x \rightarrow \pm \infty} e^{-\bar{k} x} \mathcal{I}_{1}(x)=$ $\lim _{x \rightarrow \pm \infty} e^{\bar{k} x} \mathcal{I}_{2}(x)=0$. 
From definitions (75) (76), remembering that $g\left(x^{\prime}\right)=g\left(-x^{\prime}\right)$, it is easy to verify that

$$
\mathcal{I}_{2}(x)=\mathcal{I}_{1}^{*}(-x)=\mathcal{P} \mathcal{T} \mathcal{I}_{1}(x) .
$$

The integral equation (73) allows us to compute bound state energies, too. By multiplying both sides by $h(x) e^{i b x}$ and integrating them over $x$ from $-\infty$ to $+\infty$, we obtain

$$
I_{+}=-\int_{-\infty}^{+\infty} d x h(x) e^{i b x} \int_{-\infty}^{+\infty} d x^{\prime} g\left(x^{\prime}\right) e^{i a x^{\prime}} G_{+}\left(x-x^{\prime}\right)\left(c_{S} \sigma_{z}+c_{V}\right) I_{+},
$$

or, remembering definition (28) of matrix $M_{+}$

$$
\begin{aligned}
& \left(1+\int_{-\infty}^{+\infty} d x h(x) e^{i b x} \int_{-\infty}^{+\infty} d x^{\prime} g\left(x^{\prime}\right) e^{i a x^{\prime}} G_{+}\left(x-x^{\prime}\right)\left(c_{S} \sigma_{z}+c_{V}\right)\right) I_{+} \\
\equiv & \left(\begin{array}{cc}
M_{+}^{11} & M_{+}^{12} \\
M_{+}^{21} & M_{+}^{22}
\end{array}\right)\left(\begin{array}{c}
I_{+}^{1} \\
I_{+}^{2}
\end{array}\right)=0 .
\end{aligned}
$$

Note that, since $M_{ \pm}(i \bar{k})=\mathcal{P} \mathcal{T} M_{ \pm}(i \bar{k})(\mathcal{P} \mathcal{T})^{-1}$, if $I_{ \pm}$is solution of Eq. (79), $\mathcal{P} \mathcal{T} I_{ \pm}$is solution, too.

The necessary condition for a non-trivial solution of the above equation

$$
\operatorname{det} M_{+}=1+\frac{c_{V} E+c_{S} m}{\sqrt{m^{2}-E^{2}}} S_{+}+\frac{\left(c_{V}^{2}-c_{S}^{2}\right)}{4}\left(D_{+}^{2}-S_{+}^{2}\right)=0,
$$

where $S_{+}$and $D_{+}$are functions of $k(E)$, fixes bound state energies as the roots of the equation in the interval $-m<E<+m$. Not surprisingly, bound states correspond to poles of the transmission coefficient $T_{L \rightarrow R}$ (37).

Eq. (79) allows one to express the ratio of the components of $I_{+}$in terms of $M_{+}$matrix elements. In general, one observes that $M_{+}^{22}=1+$ $i \frac{\lambda}{2}\left(c_{V}-c_{S}\right) S_{+} \neq 0$, so that one can exploit the second eq. (79), which gives $I_{+}^{2}=-\left(M_{+}^{21} / M_{+}^{22}\right) I_{+}^{1}$ and $\Psi_{b s}(x)$ can be written as

$$
\begin{gathered}
\Psi_{b s}(x)=-\frac{I_{ \pm}^{1}}{2 \bar{k}}\left\{e^{-\bar{k} x} \mathcal{I}_{1}(x)\left(\begin{array}{cc}
E+m & i \bar{k} \\
i \bar{k} & E-m
\end{array}\right)\right. \\
\left.+e^{\bar{k} x} \mathcal{I}_{2}(x)\left(\begin{array}{cc}
E+m & -i \bar{k} \\
-i \bar{k} & E-m
\end{array}\right)\right\}\left(\begin{array}{cc}
c_{V}+c_{S} & 0 \\
0 & c_{V}-c_{S}
\end{array}\right)\left(\begin{array}{cc}
1 \\
-\frac{i}{2} \frac{\left(c_{V}-c_{S}\right) D_{+}}{1+i \frac{\lambda}{2}\left(c_{V}-c_{S}\right) S_{+}}
\end{array}\right) .
\end{gathered}
$$

In the above formula, the modulus of $I_{+}^{1}$ is to be determined from normalization of the wave function $\Psi_{b s}(x)$. It is easy to check that $\Psi_{b s}(x)$ is 
eigenstate of $\mathcal{P} \mathcal{T}$, since the matrices in curly brackets are $\mathcal{P} \mathcal{T}$-symmetric, owing to relation (177) and the ratio $r=-\frac{i}{2} \frac{\left(c_{V}-c_{S}\right) D_{+}}{1+i \frac{\lambda}{2}\left(c_{V}-c_{S}\right) S_{+}}$is real. In fact, from definitions (21) 22), we see that, for $k=i \bar{k}, N_{+}^{(2)}(i \bar{k})=\left(N_{+}^{(1)}(i \bar{k})\right)^{*}$; thus, $S_{+}(i \bar{k}) \equiv N_{+}^{(1)}(i \bar{k})+N_{+}^{(2)}(i \bar{k})=2 \operatorname{Re}\left(N_{+}^{(1)}(i \bar{k})\right)$ is real, $D_{+}(i \bar{k}) \equiv$ $N_{+}^{(1)}(i \bar{k})-N_{+}^{(2)}(i \bar{k})=2 i \operatorname{Im}\left(N_{+}^{(1)}(i \bar{k})\right)$ is imaginary and $\lambda=i \bar{k} /(E+m)$ is imaginary, too, so that, as a final result, $r$ is real and $\mathcal{P} \mathcal{T} \Psi_{b s}(x)=\Psi_{b s}(x)$, if $I_{+}^{1}$ is chosen to be real.

Considering that $D_{+}$and $S_{+}$do not depend on $c_{V}$, or $c_{S}$, Eq. (80) can also be used to determine either potential strength $\left(c_{V}\right.$ or $\left.c_{S}\right)$, provided the other is fixed, in particular set to zero, in such a way to obtain a bound state at a given energy $E$ in the $(-m,+m)$ range. In this procedure, however, $\mathcal{P} \mathcal{T}$ symmetry is not automatically preserved, since Eq. (80) is of second degree in the unknown potential strength and might have a pair of complex conjugate solutions.

We have derived our expressions for bound-state wave functions starting from $\Psi_{+}(x)$, but we could, alternatively, start from $\Psi_{-}(x)$ and determine the constants $A_{-}$and $B_{-}$from the boundary conditions $\lim _{x \rightarrow \pm \infty} \Psi_{-}(x)=0$. In this case, both $A_{-}$and $B_{-}$must be different from zero because of the asymptotic behaviour of $G_{-}$and appear as the solution of a system of two homogeneous linear equations. The condition for a non-trivial solution of the system yields again the equation $\operatorname{det} M_{+}(i \bar{k})=0$, as expected, with $\operatorname{det} M_{+}$ written in terms of $\operatorname{det} M_{-}$according to formula (58).

\section{The Yamaguchi potential}

As an example of application of the formalism developed in the preceding sections, we now work out in detail a one-dimensional $\mathcal{P} \mathcal{T}$-symmetric version of the Yamaguchi potential[27], originally aimed at describing bound and scattering states of the neutron-proton system. We assume

$$
g(x)=\exp (-c|x|), h(y)=\exp (-d|y|),(-\infty<x, y<+\infty)
$$

with $c$ and $d$ positive constants, so that the Fourier transforms are

$$
\widetilde{g}(q)=\frac{2 c}{c^{2}+q^{2}}, \widetilde{h}\left(q^{\prime}\right)=\frac{2 d}{d^{2}+q^{\prime 2}} .\left(-\infty<q, q^{\prime}<+\infty\right)
$$


and the $\mathcal{P} \mathcal{T}$-symmetric kernel reads

$$
K(x, y)=e^{-c|x|+i a x} e^{-d|y|+i b y},
$$

with $a$ and $b$ real constants. With the above definitions the basic integrals $N_{ \pm}^{(1)}$ from formula (21) and $N_{ \pm}^{(2)}$ from formula (22), as well as their linear combinations $S_{ \pm}=N_{ \pm}^{(1)}+N_{ \pm}^{(2)}$ and $D_{ \pm}=N_{ \pm}^{(1)}-N_{ \pm}^{(2)}$, can be computed by elementary methods. We only quote the final results

$$
\begin{aligned}
& N_{ \pm}^{(1)}=-\frac{i}{c} \widetilde{g}(a \mp k) \frac{(a+b) c+(c+d)(a \mp k)}{(a+b)^{2}+(c+d)^{2}}+\frac{\widetilde{g}(a \mp k) \widetilde{h}(b \pm k)}{2}\left(1+i \frac{b \pm k}{d}\right), \\
& N_{ \pm}^{(2)}=\frac{i}{c} \widetilde{g}(a \pm k) \frac{(a+b) c+(c+d)(a \pm k)}{(a+b)^{2}+(c+d)^{2}}+\frac{\widetilde{g}(a \pm k) \widetilde{h}(b \mp k)}{2}\left(1-i \frac{b \mp k}{d}\right) .
\end{aligned}
$$

Formulae (86) become particularly simple when applied to the analysis of bound states: in this case, we already know from the previous section that $N_{ \pm}^{(2)}(i \bar{k})=\left(N_{ \pm}^{(1)}(i \bar{k})\right)^{*}$. Therefore, $S_{+} \equiv N_{+}^{(1)}+N_{+}^{(2)}=2 \operatorname{Re}\left(N_{+}^{(1)}\right)$ and $D_{+} \equiv N_{+}^{(1)}-N_{+}^{(2)}=2 i \operatorname{Im}\left(N_{+}^{(1)}\right)$; the left-hand-side of Eq. (801) thus becomes real in the interval $-m<E<+m$ : bound state energies are roots of the real equation

$$
\operatorname{det} M_{+}=1+\frac{2\left(c_{V} E+c_{S} m\right)}{\sqrt{m^{2}-E^{2}}} \operatorname{Re}\left(N_{+}^{(1)}\right)-\left(c_{V}^{2}-c_{S}^{2}\right)\left|N_{+}^{(1)}\right|^{2}=0
$$

If we put $c_{S}=0$ in the above equation, this allows us to derive the strength $c_{V}$ at which the purely vector potential has a bound state at given real energy $E$; in fact, Eq. (877) can be considered as a quadratic equation in $c_{V}$, with real solutions

$$
c_{V}=\frac{\frac{E R e\left(N_{+}^{(1)}\right)}{\sqrt{m^{2}-E^{2}}} \pm \sqrt{\frac{E^{2}\left(\operatorname{Re}\left(N_{+}^{(1)}\right)\right)^{2}}{m^{2}-E^{2}}+\left|N_{+}^{(1)}\right|^{2}}}{\left|N_{+}^{(1)}\right|^{2}} .
$$

Note that one of the two solutions for $c_{V}$ is always positive.

In order to complete the discussion of bound state wave functions, we give the corresponding expressions of integrals (75) and 76), obtained by 
elementary integration:

$$
\begin{aligned}
e^{-\bar{k} x} \mathcal{I}_{1}(x) & =\theta(-x) \frac{e^{(c+i a) x}}{c+\bar{k}+i a}+\theta(x)\left[\frac{e^{-\bar{k} x}}{c+\bar{k}+i a}+\frac{e^{(-c+i a) x}-e^{-\bar{k} x}}{-c+\bar{k}+i a}\right], \\
e^{\bar{k} x} \mathcal{I}_{2}(x) & =\theta(-x)\left[\frac{e^{\bar{k} x}-e^{(c+i a) x}}{c-\bar{k}+i a}+\frac{e^{\bar{k} x}}{c+\bar{k}-i a}\right]+\theta(x) \frac{e^{(-c+i a) x}}{c+\bar{k}-i a}
\end{aligned}
$$

While possible bound state wave functions with real energy are eigenstates of $\mathcal{P} \mathcal{T}$, scattering wave functions never are, but show some interesting peculiarities related to transmission resonances when $c_{V}= \pm c_{S}$, which makes it worthwhile to focus our numerical analysis on that cases. Figure 1 shows
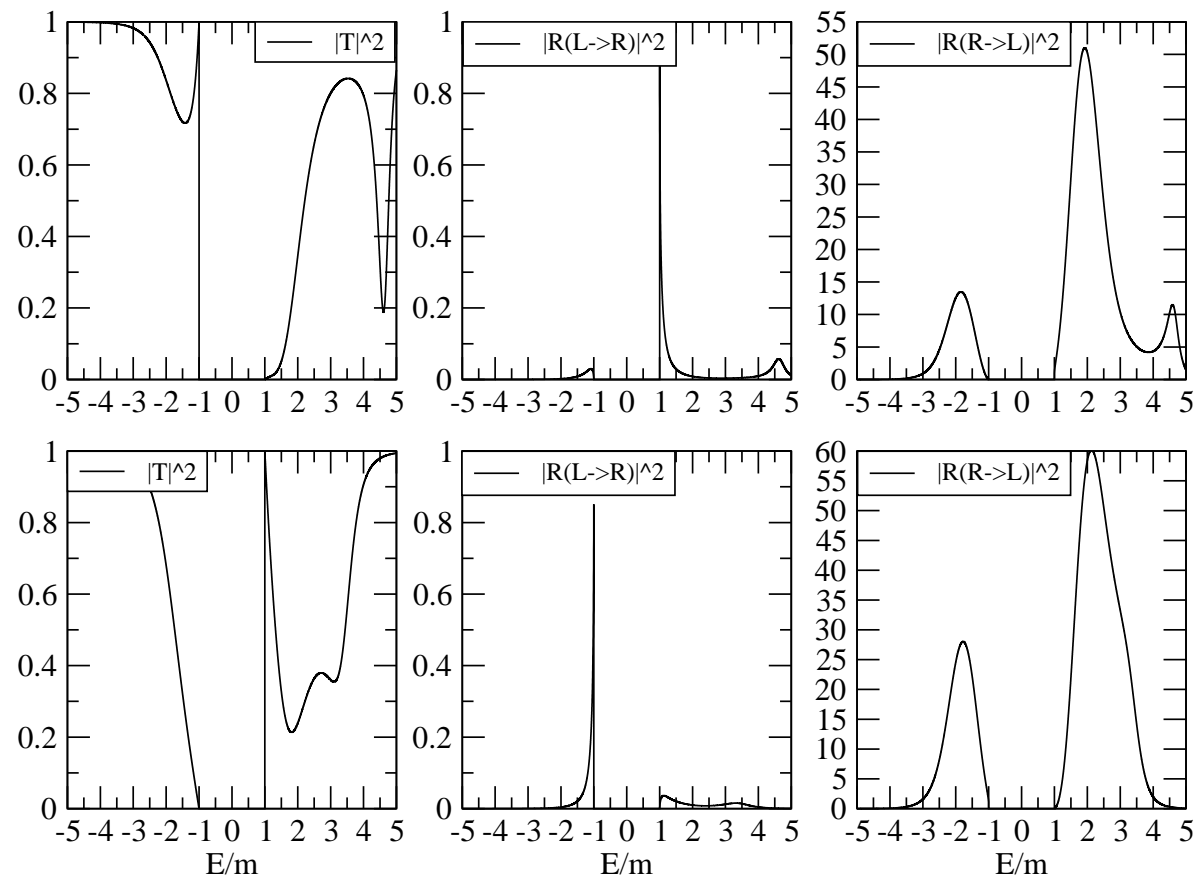

Figure 1: Square moduli of transmission and reflection coefficients vs. energy (in units of particle mass) for Yamaguchi potentials with $a=2, b=1$, $c=d=1$ and $c_{V}=c_{S}=5 \mathrm{~m}$ (upper panels), or $c_{V}=-c_{S}=5 \mathrm{~m}$ (lower panels).

the square moduli of transmission coefficients, $\left|T_{L \rightarrow R}\right|^{2}=\left|T_{R \rightarrow L}\right|^{2} \equiv|T|^{2}$, and of reflection coefficients, $\left|R_{L \rightarrow R}\right|^{2}$ and $\left|R_{R \rightarrow L}\right|^{2}$, as functions of total energy $E$ for the following choices of potential parameters: $a=2, b=1$, $c=d=1$ and $c_{V}=c_{S}=5 \mathrm{~m}$ (upper panels), or $c_{V}=-c_{S}=5 \mathrm{~m}$ (lower 
panels). $E$ ranges from $-5 m$ to $+5 m$, but the coefficients are not calculated in the $-m<E<+m$ interval, where they might have poles corresponding to bound states. When $c_{V}=c_{S}$, there is a sharp transmission resonance at $E=-m$, which appears at $E=+m$ when $c_{V}=-c_{S}$, as expected from the relation $T_{L \rightarrow R}\left(c_{V}, c_{S}, E, k\right)=T_{L \rightarrow R}\left(-c_{V}, c_{S},-E, k\right)$. These zero-energy resonances, called half-bound states, have $T_{L \rightarrow R}=T_{R \rightarrow L}=1$ and $R_{L \rightarrow R}=R_{R \rightarrow L}=0$. In both cases, the reflection coefficients show the handedness discussed in Ref.[10]: the potentials behave as absorptive for progressive waves $\left(\left|T_{L \rightarrow R}\right|^{2}+\left|R_{L \rightarrow R}\right|^{2}<1\right)$ and generative for regressive waves $\left(\left|T_{R \rightarrow L}\right|^{2}+\left|R_{R \rightarrow L}\right|^{2}>1\right)$. This pattern depends on the (common) sign of $a$ and $b$ : in fact, owing to the form (7) of the kernel, where $g(x)=e^{-c|x|}$ and $h(y)=e^{-d|y|}$ are even functions of their arguments, changing $a$ into $-a$ and $b$ into $-b$ is equivalent to a parity transformation $(x \rightarrow-x$ and $y \rightarrow-y)$, namely

$$
\begin{aligned}
T_{L \rightarrow R}(-a,-b) & =T_{R \rightarrow L}(a, b), \\
R_{L \rightarrow R}(-a,-b) & =R_{R \rightarrow L}(a, b) .
\end{aligned}
$$

In our case, with $a=-2$ and $b=-1$, the potential would become generative for progressive waves and absorptive for regressive ones. Handedness, however, is not a general rule: Figure 2 shows transmission and reflection coefficients for $a=-2, b=+1, c=d=1$ and $c_{V}=c_{S}=2 m$ (upper panels), or $c_{V}=-c_{S}=2 m$ (lower panels). In this case, both $\left|T_{L \rightarrow R}\right|^{2}+\left|R_{L \rightarrow R}\right|^{2}$ and $\left|T_{R \rightarrow L}\right|^{2}+\left|R_{R \rightarrow L}\right|^{2}$ may be $\lessgtr 1$ in different energy intervals.

As for bound states, they exist only in the lower panel cases of Figs.1,2: when $c_{V}=-c_{S}=5 m, a=2, b=1, c=d=1$ ( Fig.1 ) there is a real bound state with energy $\epsilon_{b s}=+0.3835 m$, when $c_{V}=-c_{S}=2 m, a=-2, b=1$, $c=d=1$ ( Fig.2), there is a real bound state at $\epsilon_{b s}=0.1815 \mathrm{~m}$. If $a$ and $b$ change, the bound states change their energies, but they do not disappear, unless $|a|,|b| \rightarrow+\infty$. In this latter case, the kernel $K(x, y)$ undergoes such rapid oscillations in the $x$, or $y$ directions that it becomes negligible on the average and cannot sustain bound states any more. In this limit, $|T| \rightarrow 1$ and $|R| \rightarrow 0$.

As far as bound states are concerned, the structure of Eq. (87) shows that only when $c_{V}=0 \operatorname{det} M_{+}$does not depend on $E$, but on $\bar{k}$ only, so that, if $\bar{k}_{b s}$ is a solution of $\operatorname{det} M_{+}(\bar{k})=0$, both energies $\epsilon_{b s}= \pm \sqrt{m^{2}-\bar{k}_{b s}^{2}}$ are acceptable. This is shown in Fig.3, where $\operatorname{det} M_{+}(\bar{k})=0$ is solved graphically for a scalar well of strength $c_{S}=-m, c=d=1$ and various values of $a=b$. With increasing the latter phases, the two bound state 

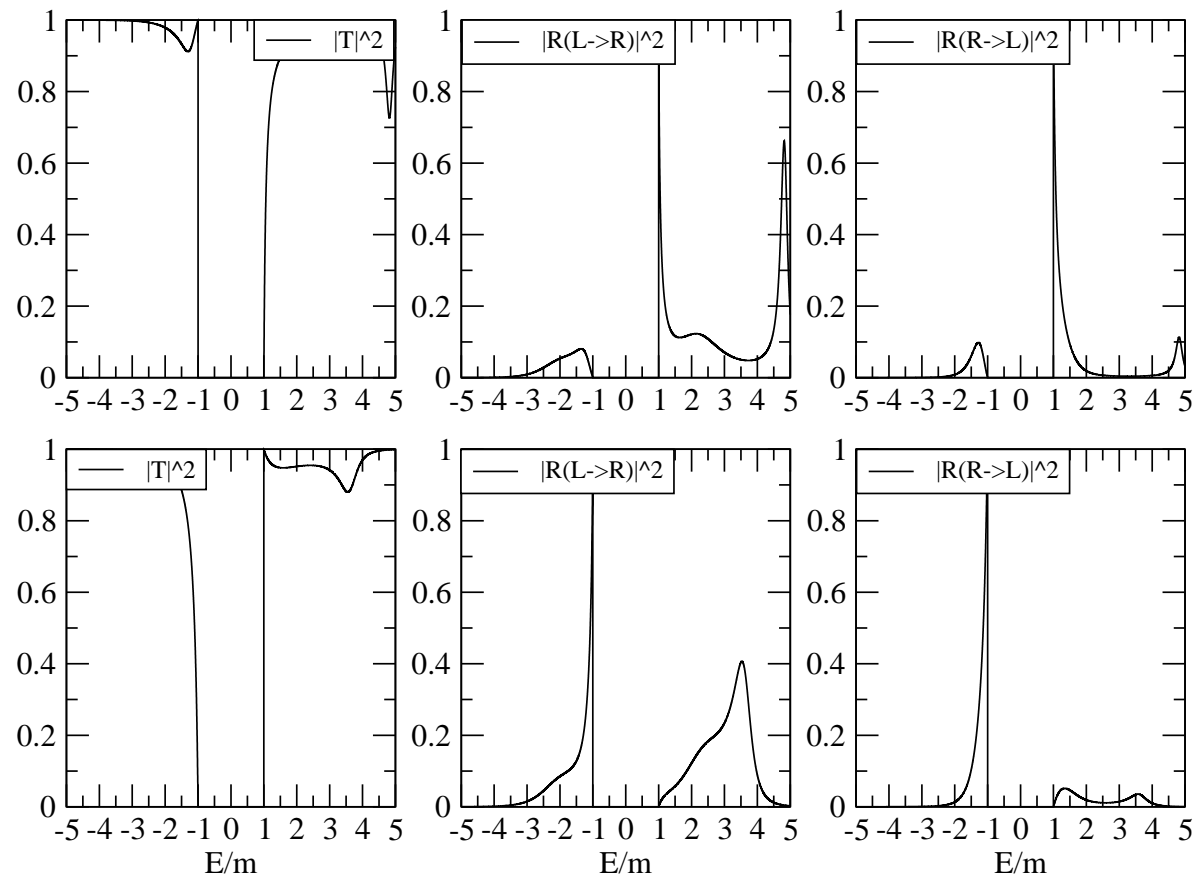

Figure 2: Square moduli of transmission and reflection coefficients vs. energy (in units of particle mass) for Yamaguchi potentials with $a=-2, b=1$, $c=d=1$ and $c_{V}=c_{S}=2 m$ (upper panels), or $c_{V}=-c_{S}=2 m$ (lower panels).

energies quickly tend to the thresholds of continuum, $\pm m$. For instance, when $a=b=10, \epsilon_{b s}= \pm 0.999999923 m$ and, in the continuum of scattering states, the potential is almost reflectionless.

\section{Conclusions and perspectives}

In this work we have studied non-local $\mathcal{P} \mathcal{T}$-symmetric potentials in the onedimensional Dirac equation. Owing to the fact that the definition of the $S$ matrix adopted in our previous work[1] dedicated to non-relativistic quantum mechanics is valid also in the relativistic case (see e.g. Ref.[28]), we have used in the present work general properties of the $S$ matrix under $\mathcal{P}, \mathcal{T}$ and $\mathcal{P} \mathcal{T}$ transformations derived in Ref.[11]. There are, of course, kinematical 


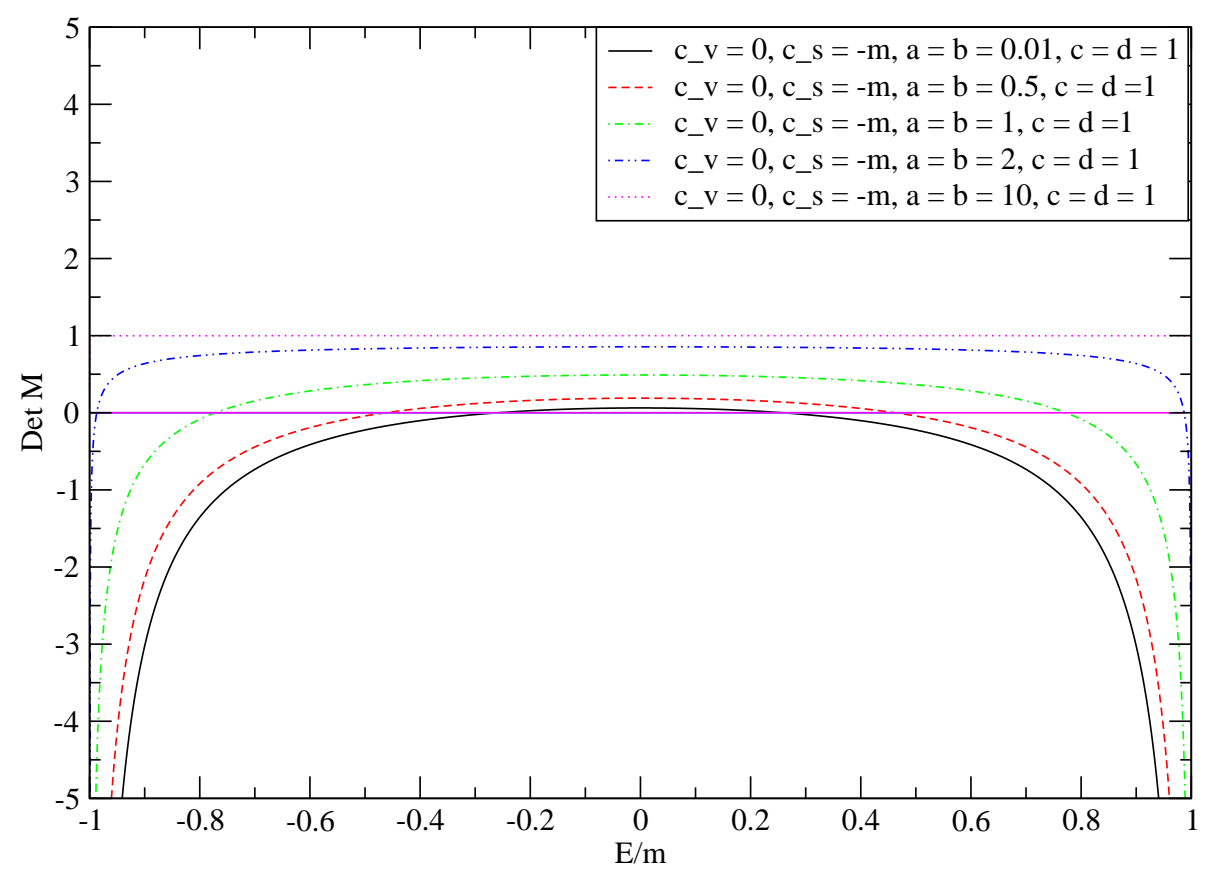

Figure 3: Denominator of the transmission coefficient for a scalar well with $c_{S}=-m, c=d=1$ at various values of $a=b$.

differences between Schrödinger and Dirac formulations: in the latter case, total energies $E$ can be either positive or negative; scattering states have either $E / m \leq-1$ or $E / m>+1$, while bound states are found in the interval $-1<E / m<+1$.

The separable potential we have studied is very flexible, since, for instance, it permits determining the real vector strength $c_{V}$ ( with scalar strength $c_{S}=0$ ) that yields a bound state at an energy $E$ arbitrarily chosen in the $[-m,+m]$ interval (see Eqs.(80,87)).

Moreover, starting from the real kernel with real coupling strengths $c_{V}$ and $c_{S}$ and $a=b=0$, one can extend it in a natural way to the generalized Hermitian case, with $g=h$ and $a=-b$, and, finally, to the $\mathcal{P} \mathcal{T}$-symmetric case, with $g$ and $h$ even functions of their arguments and arbitrary $a$ and $b$.

The specific choice of form factors $g(x)=e^{-c|x|}$ and $h(y)=e^{-d|y|}$ yields in the non-relativistic case transmission and reflection coefficients that are rational functions of momentum, $k$, since they can be written as ratios of 
polynomials in $k$. This opens the way to an algebraic search for zeros of denominators, providing information on bound states, and of numerators, e. g. of reflection coefficients ( transparency at given momentum $k$ ), or transmission coefficients ( total reflectiveness at given $k$ ).

In the relativistic case the functional dependence is more involved, due to the square root dependence on $k$ of energy $E=\sqrt{k^{2}+m^{2}}$. Nevertheless, it is interesting to remark that, in addition to the study of properties of $T$ and $R$ at given $c_{V}$ and $c_{S}$, one can study specific properties like absence of reflection or of transmission at given $k$ as functions of $c_{V}$ and $c_{S}$ : this can be easily done since transmission and reflection coefficients are, respectively, second order polynomial in $c_{V}$ and/or $c_{S}$ over second order polynomial and first order over second order.

Study of the zeros of the denominators has already been mentioned in connection with bound states. In the present work, we have made an effective approach to $\mathcal{P} \mathcal{T}$ symmetry, allowing for unitarity breaking of the scattering matrix. The search for a Hermitian equivalent description would imply the definition of a charge conjugation operator $\mathcal{C}$, in the spirit of Ref.[3], or a metric operator $\eta_{+}$, according to Ref. [6] and the study would be far from trivial. To our knowledge, $\eta_{+}$in relativistic problems involving scattering states has been exactly determined until now only for a non-Hermitian form of the Klein-Gordon equation, either free 29], or with a minimally coupled electromagnetic field [30].

This kind of more fundamental study, however, would be more appropriate to finite-range potentials with exact $\mathcal{P} \mathcal{T}$ symmetry, i.e. having a purely real discrete spectrum with eigen-functions that are eigenstates of $\mathcal{P} \mathcal{T}$ and reflectionless in the continuum, as discussed in ref.[11]. This could not be pursued for non-local potentials, but it could work for the $\mathcal{P} \mathcal{T}$-symmetric generalization of local scalar, or pseudoscalar reflectionless potentials, like those constructed in Refs. [31]-[32].

\section{A Appendix}

Here we explain the connection between the time-independent Green functions used in the present work and the time dependent ones, which are solutions to the equation

$$
\left(-i \alpha_{x} \frac{\partial}{\partial x}+\beta m-i \frac{\partial}{\partial t}\right) G\left(x, t ; x^{\prime}, t^{\prime}\right)=\delta\left(x-x^{\prime}\right) \delta\left(t-t^{\prime}\right) .
$$


We know from textbooks [33] that particular solutions to Eq. (91) are the retarded component of the causal Green function

$$
G_{\text {ret. }}^{c}\left(x, t ; x^{\prime}, t^{\prime}\right)=\theta\left(t-t^{\prime}\right) G_{+}\left(x-x^{\prime}, t-t^{\prime}\right)
$$

and the advanced component

$$
G_{a d v .}^{c}\left(x, t ; x^{\prime}, t^{\prime}\right)=\theta\left(t^{\prime}-t\right) G_{-}\left(x-x^{\prime}, t-t^{\prime}\right),
$$

where $\theta(\tau)=1$ for $\tau>0$ and $\theta(\tau)=0$ for $\tau<0$.

By inserting formulae (92,93) into Eq. (91), we obtain

$$
\begin{aligned}
& \theta\left( \pm\left(t-t^{\prime}\right)\right)\left(-i \alpha_{x} \frac{\partial}{\partial x}+\beta m-i \frac{\partial}{\partial t}\right) G_{ \pm}\left(x-x^{\prime}, t-t^{\prime}\right) \\
& +i \delta\left(t-t^{\prime}\right) G_{ \pm}\left(x-x^{\prime}, t-t^{\prime}\right)=\delta\left(x-x^{\prime}\right) \delta\left(t-t^{\prime}\right)
\end{aligned}
$$

where we have exploited the well-known relation $\frac{\partial}{\partial t} \theta\left( \pm\left(t-t^{\prime}\right)\right)=\delta\left(t-t^{\prime}\right)$. Let us multiply both sides of Eq. (94) by $\exp \left(i \mathcal{E}\left(t-t^{\prime}\right)\right)$ and integrate them over $u \equiv t-t^{\prime}$ from $-\infty$ to $+\infty$, with $\mathcal{E}$ a complex number whose imaginary part is chosen in such a way that the integral exists: we must assume $\mathcal{E}_{+}=E+i \epsilon$ for $G_{+}$and $\mathcal{E}_{-}=E-i \epsilon$ for $G_{-}$, with $\epsilon>0$. Thus, $G_{+}$ satisfies the equation

$$
\int_{0}^{+\infty} d u e^{i \mathcal{E}_{+} u}\left(-i \alpha_{x} \frac{\partial}{\partial x}+\beta m-i \frac{\partial}{\partial u}\right) G_{+}\left(x-x^{\prime}, u\right)-i G_{+}\left(x-x^{\prime}, 0\right)=\delta\left(x-x^{\prime}\right) .
$$

The above equation can be simplified by integrating by parts the third integral on the left-hand side

$$
\begin{aligned}
-i \int_{0}^{+\infty} d u e^{i \mathcal{E}_{+} u} \frac{\partial}{\partial u} G_{+}\left(x-x^{\prime}, u\right) & =-i\left|e^{i \mathcal{E}_{+} u} G_{+}\left(x-x^{\prime}, u\right)\right|_{u=0}^{u=+\infty} \\
-\mathcal{E}_{+} \int_{0}^{+\infty} d u e^{i \mathcal{E}_{+} u} G_{+}\left(x-x^{\prime}, u\right) & =i G_{+}\left(x-x^{\prime}, 0\right)-\mathcal{E}_{+} \int_{0}^{+\infty} d u e^{i \mathcal{E}_{+} u} G_{+}\left(x-x^{\prime}, u\right)
\end{aligned}
$$

and the function

$$
G_{+}\left(x-x^{\prime}\right) \equiv \int_{0}^{+\infty} d u e^{i \mathcal{E}_{+} u} G_{+}\left(x-x^{\prime}, u\right)=\int_{0}^{+\infty} d u e^{i E u-\epsilon u} G_{+}\left(x-x^{\prime}, u\right)
$$

which is nothing but the Laplace transform of $G_{+}\left(x-x^{\prime}, t-t^{\prime}\right)$ with respect to time, satisfies the equation

$$
\left(-i \alpha_{x} \frac{\partial}{\partial x}+\beta m-(E+i \epsilon)\right) G_{+}\left(x-x^{\prime}\right)=\delta\left(x-x^{\prime}\right)
$$


and can be identified with the Green function corresponding to the complex energy $E+i \epsilon$ for the time-independent Dirac equation.

We can proceed in the same way for $G_{-}\left(x-x^{\prime}, t-t^{\prime}\right)$, after introducing the complex energy $\mathcal{E}_{-}=E-i \epsilon$

$$
\int_{-\infty}^{0} d u e^{i \mathcal{E}_{-} u}\left(-i \alpha_{x} \frac{\partial}{\partial x}+\beta m-i \frac{\partial}{\partial u}\right) G_{-}\left(x-x^{\prime}, u\right)+i G_{-}\left(x-x^{\prime}, 0\right)=\delta\left(x-x^{\prime}\right) .
$$

After integrating by parts the third integral on the l.h.s. of the above equation and defining the Laplace transform with respect to time of $G_{-}\left(x-x^{\prime}, t^{\prime}-t\right)$

$$
G_{-}\left(x-x^{\prime}\right) \equiv \int_{-\infty}^{0} d u e^{i \mathcal{E}_{-} u} G_{-}\left(x-x^{\prime}, u\right)=\int_{0}^{+\infty} d v e^{-i E v-\epsilon v} G_{-}\left(x-x^{\prime},-v\right)
$$

we arrive at the equation satisfied by the time-independent Green function $G_{-}\left(x-x^{\prime}\right)$

$$
\left(-i \alpha_{x} \frac{\partial}{\partial x}+\beta m-(E-i \epsilon)\right) G_{-}\left(x-x^{\prime}\right)=\delta\left(x-x^{\prime}\right) .
$$

Eqs. (97,100) coincide with Eq. (8) of the text.

\section{B Appendix}

Formula (58) can be easily proved from definition (30), according to which

$$
\begin{gathered}
\operatorname{det} M_{+}-\operatorname{det} M_{-}=i \frac{c_{V} E+c_{S} m}{k}\left(S_{+}+S_{-}\right)+\frac{c_{V}^{2}-c_{S}^{2}}{4}\left(D_{+}^{2}-D_{-}^{2}-S_{+}^{2}+S_{-}^{2}\right) \\
=i \frac{c_{V} E+c_{S} m}{k}\left(N_{+}^{(1)}+N_{+}^{(2)}+N_{-}^{(1)}+N_{-}^{(2)}\right)+\left(c_{V}^{2}-c_{S}^{2}\right)\left(N_{-}^{(1)} N_{-}^{(2)}-N_{+}^{(1)} N_{+}^{(2)}\right),
\end{gathered}
$$

where integrals $N_{ \pm}^{(1)}$ and $N_{ \pm}^{(2)}$ are defined by formulae (21) and (22) respectively. We promptly obtain from the definitions

$$
\begin{gathered}
N_{+}^{(1)}+N_{+}^{(2)}+N_{-}^{(1)}+N_{-}^{(2)}=2 \int_{-\infty}^{+\infty} d x h(x) e^{i b x} \int_{-\infty}^{+\infty} d x^{\prime} g\left(x^{\prime}\right) e^{i a x^{\prime}} \cos \left(k\left(x-x^{\prime}\right)\right) \theta\left(x-x^{\prime}\right) \\
+2 \int_{-\infty}^{+\infty} d x h(x) e^{i b x} \int_{-\infty}^{+\infty} d x^{\prime} g\left(x^{\prime}\right) e^{i a x^{\prime}} \cos \left(k\left(x-x^{\prime}\right)\right) \theta\left(x^{\prime}-x\right) \\
=\int_{-\infty}^{+\infty} d x h(x) e^{i b x} \int_{-\infty}^{+\infty} d x^{\prime} g\left(x^{\prime}\right) e^{i a x^{\prime}}\left(e^{i k\left(x-x^{\prime}\right)}+e^{-i k\left(x-x^{\prime}\right)}\right) \\
=\widetilde{g}(a-k) \widetilde{h}(b+k)+\widetilde{g}(a+k) \widetilde{h}(b-k)
\end{gathered}
$$


and

$$
\begin{gathered}
N_{-}^{(1)} N_{-}^{(2)}-N_{+}^{(1)} N_{+}^{(2)}=-\widetilde{g}(a-k) \widetilde{h}(b+k) \widetilde{g}(a+k) \widetilde{h}(b-k) \\
+\widetilde{g}(a-k) \widetilde{h}(b+k) N_{-}^{(1)}+\widetilde{g}(a+k) \widetilde{h}(b-k) N_{-}^{(2)} .
\end{gathered}
$$

In deriving the last expression, the relation $\theta(-x)=1-\theta(x)$ has been used in the integrands. Inserting the right-hand sides of formulae (102, 103) into formula (101) yields formula (58) of the text.

It is worthwhile to point out that the definitions we have used and, consequently, the relation between $\operatorname{det} M_{+}$and $\operatorname{det} M_{-}$are also valid for complex $k$, in particular for $k=i \bar{k}$, with $\bar{k}>0$, characterizing bound states with real energy.

\section{References}

[1] Bender C M and Boettcher S 1998 Phys. Rev. Lett. 805243.

[2] Bender C M, Boettcher S and Meisinger P N 1999 J. Math. Phys. 40 2201.

[3] Bender C M 2007 Rep. Prog. Phys. 70947.

[4] Scholtz F G, Geyer H B and Hahne F J W 1992 Ann. Phys. 21374.

[5] Mostafazadeh A 2002 J. Math. Phys. 43205.

[6] Mostafazadeh A 2008 J. Phys.A: Math. Theor. 41244017.

[7] Jones H F 2007 Phys. Rev. D 76125003.

[8] Znojil M 2008 Phys. Rev. D 78025026.

[9] Znojil M 2008 J. Phys. A : Math. Theor. 41292002.

[10] Ahmed Z 2004 Phys. Lett. A 324152.

[11] Cannata F, Dedonder J-P and Ventura A 2007 Ann. Phys. 322397.

[12] Cannata F and Ventura A 2006 Czech. J. Phys. 56943.

[13] Sinha A and Roy P 2005 Mod. Phys. Lett. A 202377. 
[14] Sinha A and Roy P 2006 J. Phys. A : Math. Gen. 39 L377.

[15] Egrifes H and Sever R 2005 Phys. Lett. A 344117.

[16] Jia C S and de Souza Dutra A 2006 J. Phys. A : Math. Gen. 3911877.

[17] Jia C S, Liu J Y, Wang P Q and Che C S 2007 Phys. Lett. A 369274.

[18] Jia C S, Diao Y F and Liu J Y 2008 Int. J. Theor. Phys. 47664.

[19] Jia C S and de Souza Dutra A 2008 Ann. Phys. 323566.

[20] Cannata F and Ventura A 2008 Phys. Lett. A 372941.

[21] McKellar B H J and Stephenson Jr G J 1987 Phys. Rev. C 352262.

[22] Calkin M G, Kiang D and Nogami Y 1988 Phys. Rev. C 381076.

[23] Dominguez-Adame F and González M A 1992 Physica B 176180.

[24] Bell J S and Ruegg H 1975 Nucl. Phys. B 98151.

[25] Ginocchio J N 1997 Phys. Rev. Lett. 78436.

[26] Ginocchio J N 2005 Phys. Rep. 414165 and references therein.

[27] Yamaguchi Y 1954 Phys. Rev. 951628.

[28] Weinberg S 1995 The Quantum Theory of Fields, Vol. I: Foundations (Cambridge University Press, New York) Ch.3.

[29] Mostafazadeh A 2003 Class. Quantum Grav. 20155.

[30] Mostafazadeh A and Zamani F 2006 Ann. Phys. 3212183.

[31] Toyama F M, Nogami Y and Zhao Z 1993 Phys. Rev. A 47897.

[32] Nogami Y and Toyama F M 1998 Phys. Rev. A 5793.

[33] Bogoliubov N N and Shirkov D V 1959 Introduction to the Theory of Quantized Fields (Interscience Publishers, New York) Ch. 14. 